ARTICLE

\title{
A Brainstem reticulotegmental neural ensemble drives acoustic startle reflexes
}

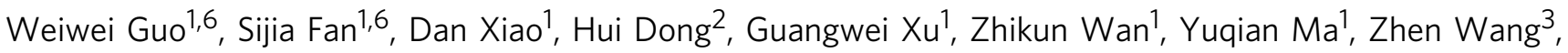
Tian Xue ${ }^{1,4}$, Yifeng Zhou', Yulong Li i[ ${ }^{2,5} \&$ Wei Xiong (i] ${ }^{1,4 凶}$

The reticulotegmental nucleus (RtTg) has long been recognized as a crucial component of brainstem reticular formation (RF). However, the function of RtTg and its related circuits remain elusive. Here, we report a role of the RtTg in startle reflex, a highly conserved innate defensive behaviour. Optogenetic activation of RtTg neurons evokes robust startle responses in mice. The glutamatergic neurons in the RtTg are significantly activated during acoustic startle reflexes (ASR). Chemogenetic inhibition of the RtTg glutamatergic neurons decreases the ASR amplitudes. Viral tracing reveals an ASR neural circuit that the cochlear nucleus carrying auditory information sends direct excitatory innervations to the RtTg glutamatergic neurons, which in turn project to spinal motor neurons. Together, our findings describe a functional role of RtTg and its related neural circuit in startle reflexes, and demonstrate how the RF connects auditory system with motor functions.

\footnotetext{
${ }^{1}$ Institute on Aging and Brain Disorders, The First Affiliated Hospital of USTC, Division of Life Sciences and Medicine, Hefei National Laboratory for Physical Sciences at the Microscale, University of Science and Technology of China, Hefei 230026, China. ${ }^{2}$ State Key Laboratory of Membrane Biology, Peking University School of Life Sciences, Beijing 100871, China. ${ }^{3}$ Shanghai Mental Health Center, Shanghai Jiao Tong University School of Medicine, Shanghai, P.R. China. ${ }^{4}$ Center for Excellence in Brain Science and Intelligence Technology, Chinese Academy of Sciences, Shanghai 200031, China. ${ }^{5}$ PKU-IDG-McGovern Institute for Brain Research, Beijing 100871, China. ${ }^{6}$ These authors contributed equally: Weiwei Guo, Sijia Fan. $\varpi_{\text {email: wxiong@ustc.edu.cn }}$
} 
ocated in the brainstem, the reticular formation (RF) has long been recognized as one of the most important part of the central nervous system (CNS). As its name suggested, the RF resembles a net-like structure composed of numerous ascending and descending nerve fibers along with various heterogeneous nuclei extending from the spinal cord to the thalamus ${ }^{1}$. This widespread projections allow the RF to act as a "command center" of the CNS. The midbrain RF together with its ascending tracts - also known as the reticular activating system (RAS) - project diffusely to the cerebellum, thalamus, cerebral cortex, and limbic system, functioning to promote the sleep-wake cycle, arousal, and consciousness 2,3 . Lesions to RAS result in impaired consciousness and even coma in some patients ${ }^{4,5}$. Descending fibers from the RF, mainly reticulospinal tracts, project to the spinal cord to coordinate respiratory control, pain perception, posture maintenance, and muscular reflexes ${ }^{6-8}$.

In mammals, the RF comprises over 100 individual nuclei. Most of the nuclei embedded in this structure have no precise cytoarchitectural boundaries ${ }^{1}$, rendering functional studies of these RF nuclei a challenging task. Situated at the ventral extreme of the pontine tegmentum, the reticulotegmental nucleus ( $\mathrm{RtTg}$ ) is one of the least investigated neural ensembles with clear delineations of territory in $\mathrm{RF}^{9}$. Early studies in primates indicated that RtTg might be involved with the oculomotor circuits crucial for smooth-pursuit eye movement ${ }^{10}$. On the other hand, controversial conclusions have been demonstrated regarding to the functional relationship between RtTg and locomotion. Unit recordings in monkeys and cats have revealed increased RtTg neuronal activities during limb movements ${ }^{11,12}$. Another early study in mice also observed consistent activation of RtTg during motor seizure ${ }^{13}$. Still, other researchers have observed increased forward locomotion in rats following electronic lesion of the RtTg ${ }^{14}$.

Although sporadic studies on RtTg have been reported over the past few decades, the precise functional role of the RtTg and its brain-wide upstream and downstream projections have not been investigated thoroughly. Recently, the development of cell-typespecific viral tracing approaches as well as optogenetic/chemogenetic tools has enabled more accurate discovery and elucidation of the potential roles and circuit connections in a target brain region ${ }^{15}$. Using these cutting-edge techniques combined with in vitro/in vivo electrophysiological recordings, we surprisingly found that the RtTg controls a conservative startle response, and identified a neural circuit from the cochlear nucleus $(\mathrm{CN})$ to spinal motor neurons that underlies this innate defensive behaviour.

\section{Results}

Optogenetic activation of RtTg neurons elicits startle reflexes. We first conducted optogenetic experiments ${ }^{16}$ to examine the behavioural readout for RtTg neuronal activation. We unilaterally injected adeno-associated viruses (AAV) expressing Channelrhodopsin-2 (AAV-hSyn-ChR2-EGFP) into the RtTg of mice, followed by implantation of an optical fiber above the injection site (Fig. 1a). Mice transfected with AAV-hSyn-EGFP were used as controls. Brain slice patch-clamp recordings showed that a light-pulse train triggered action potentials in the $\mathrm{RtTg}$ ChR2 ${ }^{+}$neurons, indicating the effectiveness of $\mathrm{RtTg}$ neuron activation (Fig. 1b, c). Strikingly, optogenetic activation of RtTg neurons reliably evoked robust startle reflexes, which were defined as involuntarily innate defensive response occurring rapidly (often in milliseconds) after the onset of aversive stimuli (Fig. 1d-f and Supplementary Movie 1). Moreover, the startle amplitudes were positively correlated to the laser intensities (Fig. 1g). As a control, no startle reflex was observed upon photostimulation of RtTg neurons expressing EGFP only
(Fig. 1f, g and Supplementary Movie 2). It is worth noting that the optogenetic manipulation was strictly limited to the RtTg region without influencing the adjacent caudal pontine reticular nucleus (PnC) (Supplementary Fig. 1), which has been already recognized to be critical for startle reflex by previous studies $^{17-19}$.

Previous reports demonstrated that through all mammals, the stereotypical startle pattern consists of a fast contraction of neck, facial and skeletal muscles ${ }^{17}$. We therefore examined the electromyographic (EMG) activities in the neck and hindlimb of mice during optogenetic activation of RtTg neurons. As expected, we consistently detected robust EMG activities in both neck and hindlimb muscles following every single optogenetic stimulation pulse (Fig. 1h, i and Supplementary Fig. 2a, b). The EMG activities occurred very shortly after optogenetic activation of RtTg neurons, with a mean latency of $5.75 \pm 0.38 \mathrm{~ms}$ for neck muscle (Supplementary Fig. 2c) and $9.43 \pm 0.12 \mathrm{~ms}$ for hindlimb muscle (Fig. 1j) respectively.

RtTg inactivation specifically suppresses acoustic startle reflexes. We next used chemogenetic suppression-based loss-offunction experiments to further verify the potential role of RtTg in acoustic startle reflex (ASR), a major form of startle behaviours. Specifically, the AAV virus expressing mCherry-tagged hM4 $4 \mathrm{Di}^{20}$ - an inhibitory designer receptor exclusively activated by the designer drug (DREADD) - was bilaterally injected into the RtTg (Fig. 2a). We then employed a well-established paradigm $^{21}$ to trigger and measure the ASR in mice. Transient suppression of RtTg neurons by intraperitoneal (i.p.) injection of clozapine-N-oxide (CNO) significantly decreased ASR amplitudes (Fig. 2b) without affecting other innate behaviours including motor coordination (Fig. $2 \mathrm{c}-\mathrm{h}$ ) and pain perception (Fig. 2i).

We then examined the effects of Caspase3-based ablation ${ }^{22}$ of RtTg neurons on ASR. Specifically, a mixture of AAV-hSyn-Cre and AAV-flex-taCasp3-TEVp was bilaterally co-injected into the RtTg (Supplementary Fig. 3a). Immunohistochemical analysis showed a remarkable reduction in the number of cells stained positive for neuronal nuclear antigen $\left(\mathrm{NeuN}^{+}\right)$in the targeted RtTg region, confirming efficient ablation (Supplementary Fig. 3b-f). Compared with the control group, mice with RtTg lesions exhibited significantly decreased ASR amplitude (Supplementary Fig. 3g). In contrast, the RtTg ablation did not affect motor coordination (Supplementary Fig. 3h-m), arousal (Supplementary Fig. 3n), and pain perception (Supplementary Fig. 3o). Together, these results suggest that the RtTg plays an essential role specifically in startle behaviours.

The activities of RtTg glutamatergic neurons augment during ASR. We next examined the expression of c-fos, a marker for recent neural activity ${ }^{23}$, in the RtTg of mice exposed to $95 \mathrm{~dB}$ acoustic stimuli. In addition to several brain nuclei with wellcharacterized functional involvement in the central auditory system - including the $\mathrm{CN}$ and the auditory cortex $(\mathrm{AuC})^{24}-$ we also observed robust acoustic-stimuli-induced activation of RtTg neurons (Supplementary Fig. 4 and Fig. 3a, b). Pursuing this, we generated a clear map of the c-fos expression pattern of RtTg during ASR by collecting all consecutive coronal sections covering the entire RtTg area. Quantification analysis revealed a greater density of $\mathrm{c}$-fos ${ }^{+}$cells in the rostral and medial parts of the RtTg (Fig. 3c and Supplementary Fig. 5). A non-acoustic stimulus such as air puff can also elicit startle reflexes in rodents ${ }^{25}$. However, we did not detect any changed $\mathrm{c}$-fos expression in the RtTg of mice following air puff-induced startle reflexes (Supplementary Fig. 6). 


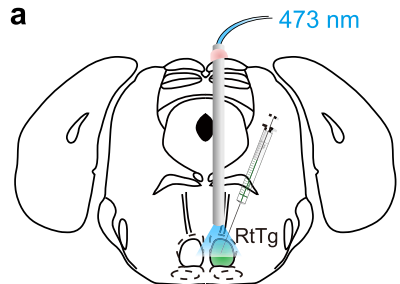

AAV-hSyn-ChR2-EGFP

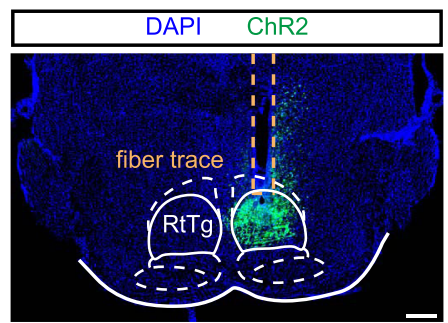

e

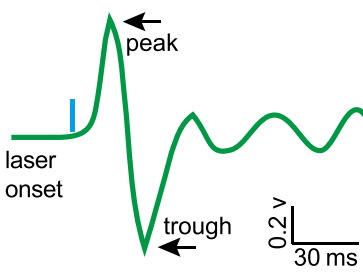

b

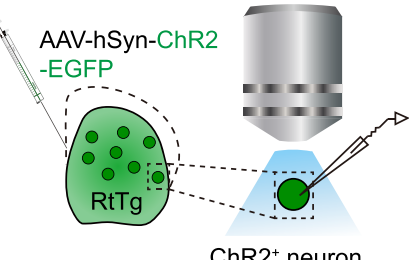

$\mathrm{ChR}^{+}$neuron
C $\quad$ RtTg ChR2+ neuron

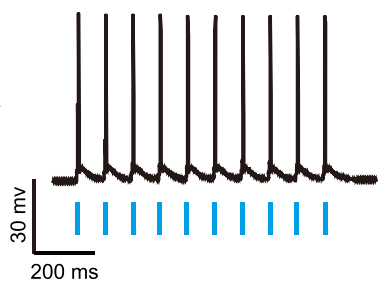

d

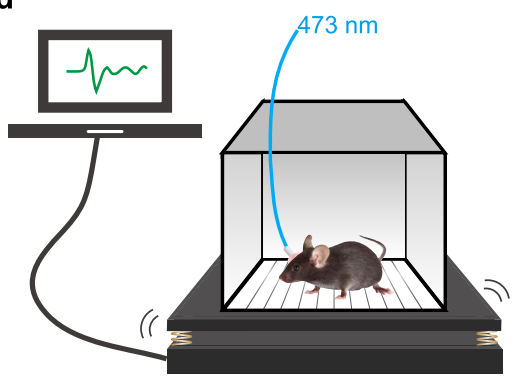

$\mathbf{f}$

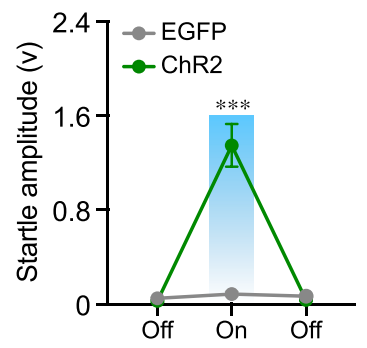

g

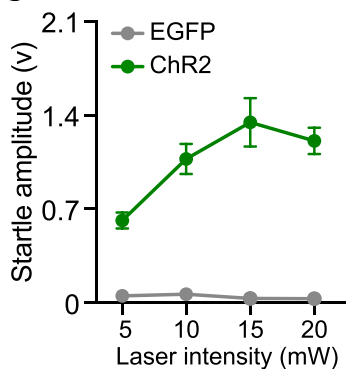

h

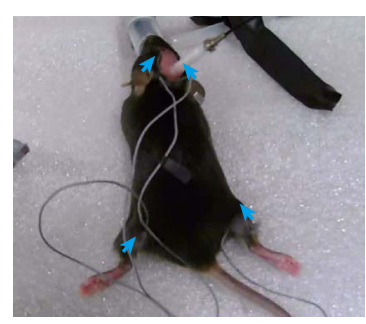

i 1.57 Hindlimb ChR2
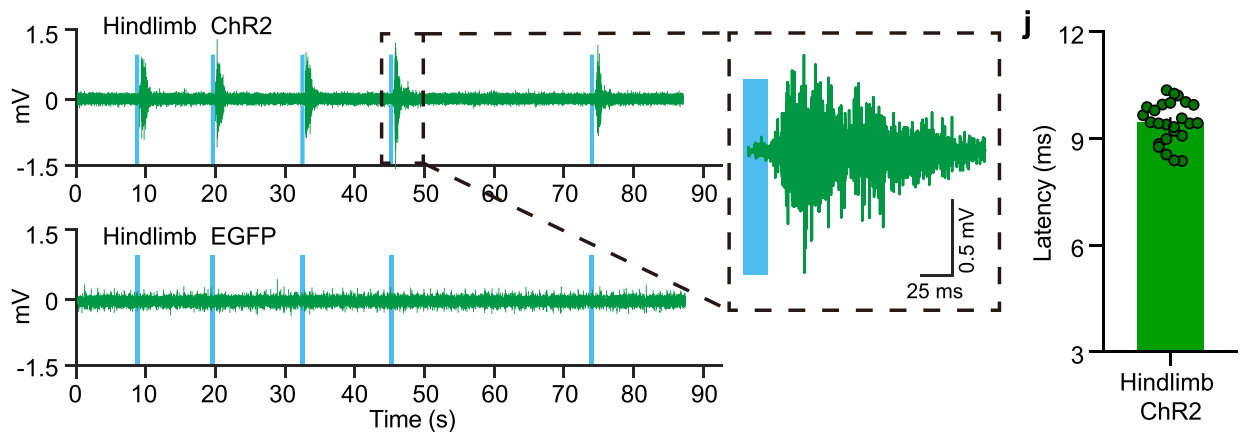

Fig. 1 Optogenetic activation of RtTg leads to stereotypical startle behaviour. a Left: schematic for optogenetic activation of the RtTg. Right: a representative image confirming ChR2-EGFP expression in the RtTg, and the optical fiber trace. Scale bar, $400 \mu \mathrm{m}$. $\mathbf{b}$ Schematic for the optogenetic activation and the simultaneous whole-cell patch-clamp recording of RtTg EGFP+ neurons in the RtTg brain slice of mice receiving an intra-RtTg injection of the AAV-hSyn-ChR2-EGFP. c Sample traces showing the photostimulation (blue bars, $1 \mathrm{~ms}$ )-evoked action potentials in RtTg EGFP+ neurons. $\mathbf{d}$ Schematic for the optogenetic activation of the RtTg and simultaneous detection of startle reflexes. e Startle amplitude was defined as the largest peak-to-peak response occurred within $200 \mathrm{~ms}$ after the onset of the laser stimulus. f Startle amplitudes evoked by opto-stimulation (blue bars) of the RtTg neurons expressing ChR2 or EGFP ( $n=15$ mice per group, two-sided unpaired $t$-tests, $P=5.78 \times 10^{-6}$ ). $\mathbf{g}$ Startle amplitudes at different laser intensities in the ChR2 and EGFP group ( $n=15$ mice per group). $\mathbf{h}$ Representative image showing the experimental setup for EMG recording during optogenetic activation of RtTg neurons. Blue arrows indicate positions for electrode insertion. $\mathbf{i}$ Example of the entire and locally amplified EMG traces showing activities of mouse hindlimb muscles during optogenetic activation (blue bar) of RtTg neurons. $\mathbf{j}$ Quantification of latency for hindlimb muscle EMG activities after optogenetic activation of the RtTg ( $n=5$ mice, 25 bouts in total). Error bar represent mean \pm s.e.m. ${ }^{\star \star \star} P<0.001$.

To further examine whether RtTg neurons are selectively responsive to strong stimuli that drive the startle or just generally sensitive to auditory cues, we performed RtTg c-fos staining after mice were introduced to white noise stimuli with weaker intensities $(45,50,55,60 \mathrm{~dB})$. These weak stimuli have been shown to be audible in mice ${ }^{26}$, but were insufficient to elicit startle reflex (Supplementary Fig. 7). As shown in Supplementary Fig. 8a, the c-fos expression level of RtTg under $45-60 \mathrm{~dB}$ noise stimuli was extremely low and had no significant correlation with these weak white noise intensities. In comparison, the RtTg total c-fos ${ }^{+}$cell number was well correlated with startle-eliciting white noise intensities (Supplementary Fig. 8b) as well as startle amplitudes (Supplementary Fig. 8c). These results demonstrate that the activity of RtTg indeed correlates with the startle response.

Screening via the Allen Brain Database indicated that RtTg neurons feature a high expression of vesicular glutamate transporter 2 (VGLUT2) mRNA, a classic marker for excitatory glutamatergic neurons. Consistently, our immunostaining results revealed that nearly $90 \%$ of c-fos ${ }^{+}$RtTg neurons co-labelled with glutamate (Fig. 3d), confirming the excitatory nature of these ASR-activated RtTg neurons.

To further monitor the dynamics of RtTg glutamatergic neurons during ASR, we injected a genetically encoded Credependent calcium indicator (AAV-DIO-Ef1a-GCamp6m) into the RtTg of Vglut2-Cre mice ${ }^{27}$ and performed fiber photometry ${ }^{28}$ (Fig. 3e). Mice transfected with AAV-DIO-Ef1a-EGFP were used as controls. Fluorescent signal fluctuations were recorded simultaneously with ASR data. As with our initial observations based on c-fos experiments, there were significantly elevated calcium signals in RtTg glutamatergic neurons upon the onset of $95 \mathrm{~dB}$ stimuli (Fig. 3f-i), whereas no signal changes were detected in control mice expressing EGFP (Supplementary Fig. 9 and Fig. 3j). The changes in calcium signals of RtTg glutamatergic neurons exhibited a positive correlation with the acoustic stimulus intensities (Supplementary Fig. 10). In contrast, we did not detect 
a

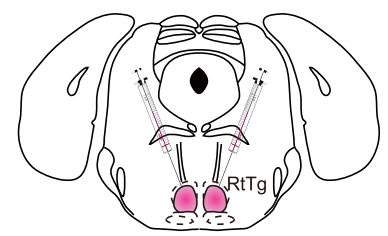

AAV-hSyn-hM4Di-mCherry

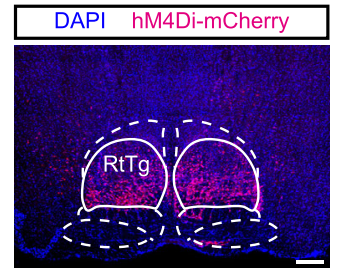

C

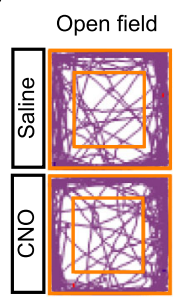

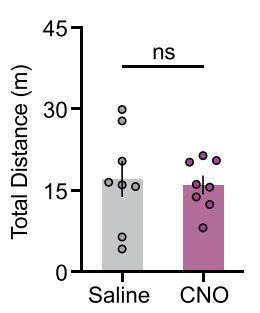

g

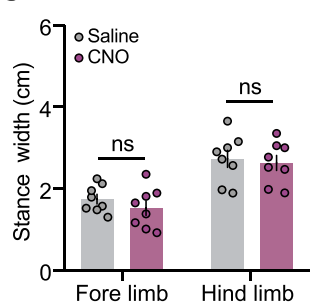

b
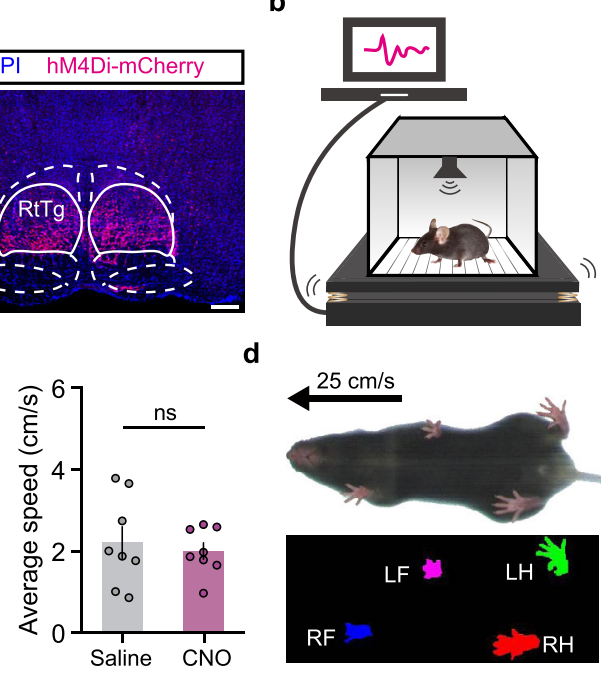

$\stackrel{25 \mathrm{~cm} / \mathrm{s}}{\longleftarrow}$

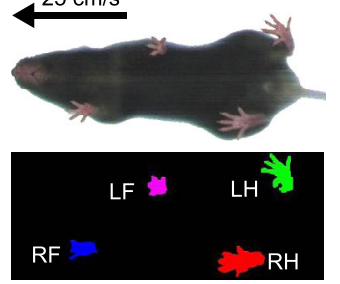

h

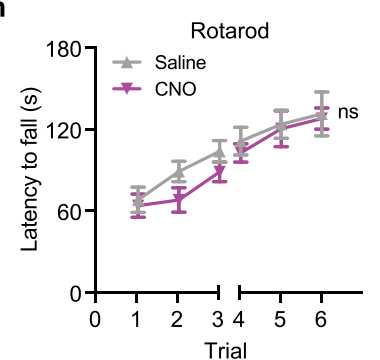

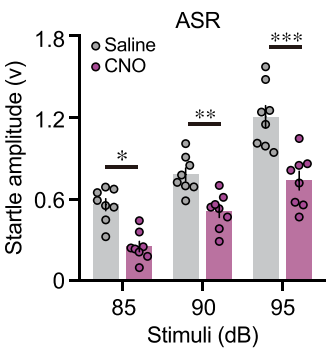

e

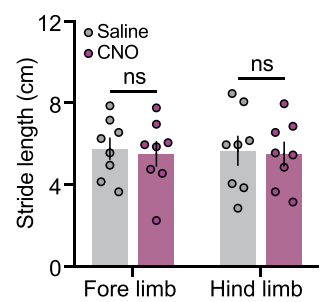

f

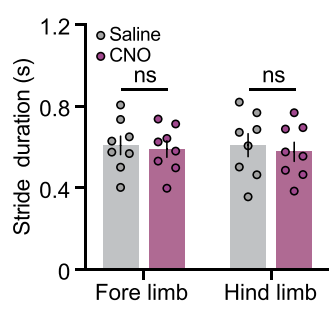

i

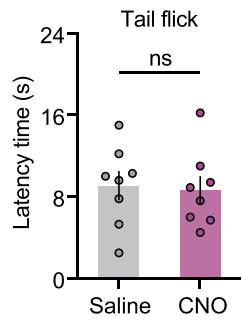

Fig. 2 Behavioural consequences of RtTg inactivation. a Left: schematic for chemogenetic inactivation of RtTg neurons. Right: a representative image confirming hM4Di-mCherry expression. Scale bar, $300 \mu \mathrm{m}$. b Left: schematic for the ASR paradigm. Right: quantitative graph showing the effect of RtTg inactivation on ASR amplitudes ( $n=8$ per group; $t=2.417, P=0.02(85 \mathrm{~dB}) ; t=3.429, P=0.0014(90 \mathrm{~dB}) ; t=5.976, P=4.3 \times 10^{-7}(95 \mathrm{~dB})$ ). c Left: representative movement tracks of hM4Di-infected mice after i.p. injection of saline or CNO. Quantification of total distances (middle, $n=8$ per group; $P=0.7637$ ) and average speed (right, $n=8$ per group; $P=0.6411$ ) during open field test. $\mathbf{d}$ Representative images showing a mouse walking on treadmill and paw prints detected from a video settled under the treadmill. e-g Digi Gait analysis for hM4Di infected mice after i.p. injection of saline or CNO ( $n=8$ per group; for e: $t=0.2945, P=0.7705$ (fore limb); $t=0.1914, P=0.8496$ (hindlimb); for $\mathrm{f}: t=0.2689, P=0.7899$ (fore limb); $t=0.4781, P=0.6363$ (hindlimb); for g: $t=0.9117, P=0.3697$ (fore limb); $t=0.4126, P=0.6831$ (hindlimb)). $\mathbf{h}$ Latency to fall on accelerating rotarod of hM4Di-infected mice after i.p. injection of saline or CNO ( $n=8$ per group; $F=0.2, P=0.9346$ ). $\mathbf{i}$ Tail-flick latency of hM4Di-infected mice after i.p. injection of saline or CNO ( $n=8$ per group, $P=0.8269$ ). Error bar represent mean \pm s.e.m. Significance was assessed using two-sided unpaired $t$-tests in $c, i$, and two-way ANOVA combining with FDR corrections in $\mathbf{b}$, e-h. ${ }^{\star} P<0.05$; ${ }^{\star \star} P<0.01$; ${ }^{\star \star \star} P<0.001$; ns, not significant.

increased calcium signals of RtTg glutamatergic neurons after the onset of air puff stimulations (Supplementary Fig. 11).

Given that startle reflex occurs rapidly in response to abrupt aversive stimuli, we next performed in vivo recording of RtTg neurons during ASR to determine whether RtTg neurons also response with short latency compatible with the initiation of the startle reflex. Mice were introduced to white noise stimuli followed by sampling the RtTg neuronal firing rate of multiple units. RtTg neuronal activity increased significantly upon the onset of acoustic stimulus with a mean spike latency of $5.12 \pm 0.47 \mathrm{~ms}$ (Fig. 3k). Furthermore, we observed a gradual increase in firing rates with elevated stimulus intensities (Supplementary Fig. 12). These results indicate that RtTg neurons can quickly response to acoustic stimuli.

Chemogenetic inhibition of RtTg glutamatergic neurons suppresses ASR. We further examined whether chemogenetic inhibition of RtTg glutamatergic neurons could reduce ASR amplitudes. A Cre-dependent AAV expressing mCherry-tagged hM4Di was bilaterally injected into the RtTg of Vglut2-Cre mice (Fig. 31 and Supplementary Fig. 13). In acute RtTg brain slices, bath application of $\mathrm{CNO}$ reduced the membrane potential as well as the firing frequency of $\mathrm{hM}_{4} \mathrm{Di}^{+} \mathrm{RtTg}$ neurons, confirming the efficacy of hM4Di- mediated inhibition (Supplementary Fig. 14). Furthermore, i.p. injected CNO significantly decreased ASR amplitudes (Fig. $3 \mathrm{~m}$ ). Note that these effects cannot be directly attributed to $\mathrm{CNO}$ or its metabolites, as the mCherry control mice injected with $\mathrm{CNO}$ exhibited no such decrease in startle response (Fig. 3n). Taken together, these results indicate that the activity of RtTg glutamatergic neurons is capable of, and necessary for driving startle reflexes.

Chemogenetic inhibition of RtTg GABAergic neurons enhances ASR. According to the Allen Brain database, neurons in the central region of the RtTg are predominantly glutamatergic, whereas neurons expressing high levels of mRNA transcripts for inhibitory GABAergic neuron marker, vesicular GABA transporter $(V G A T)$, were mainly distributed in the pericentral region of the RtTg (Supplementary Fig. 15a). Consistently, in this study, immunostaining of the brain sections from GAD1-EGFP transgenic mice ${ }^{29}$ further demonstrated the shell-distribution pattern of the inhibitory neurons embracing glutamatergic neurons in the central RtTg (Supplementary Fig. 15b). Moreover, chemogenetic inhibition of the pericentral RtTg GABAergic neurons in VgatCre mice resulted in significantly enhanced ASR amplitudes, suggesting a negative regulatory role of RtTg GABAergic neurons in startle reflexes (Supplementary Fig. 15c, d). 


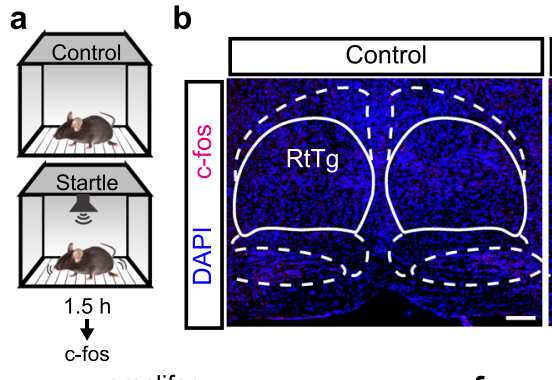

$\mathbf{e}$
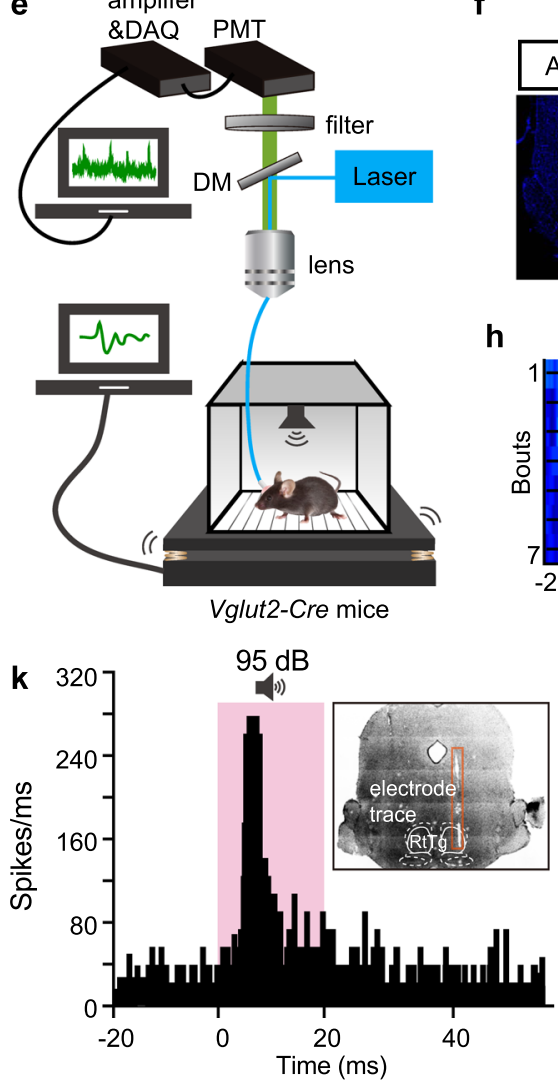

h

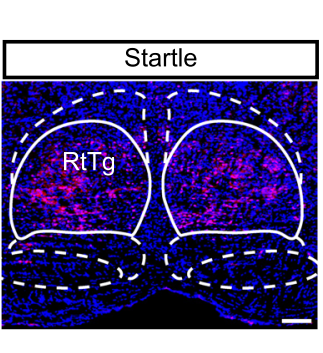

C

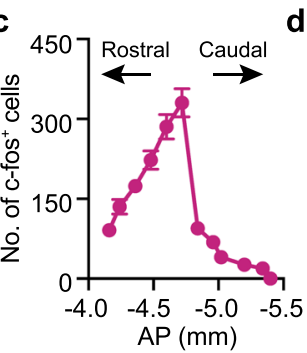

g
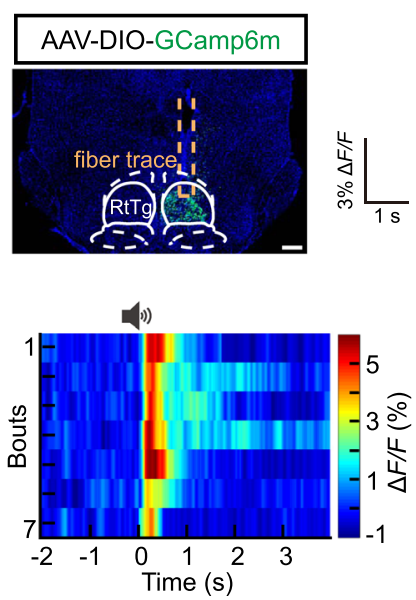

1

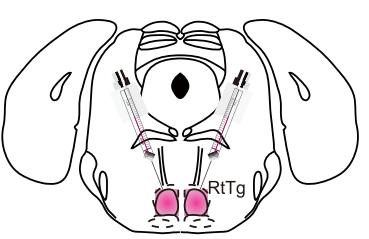

Vglut2-Cre mice

AAV-DIO-hM4Di-mCherry

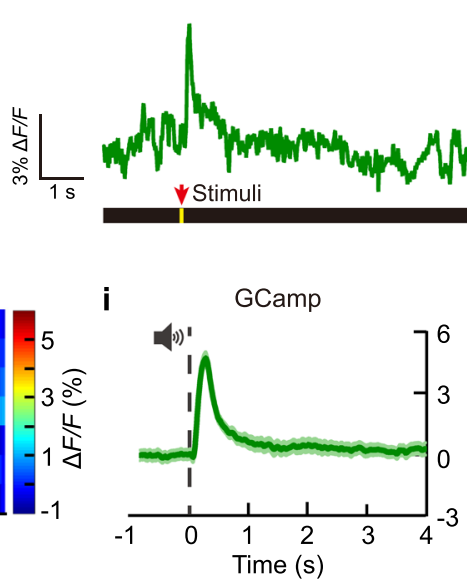

RtTg GCamp
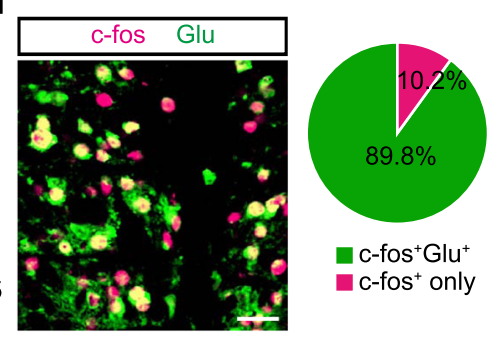

c-fos ${ }^{+}$only
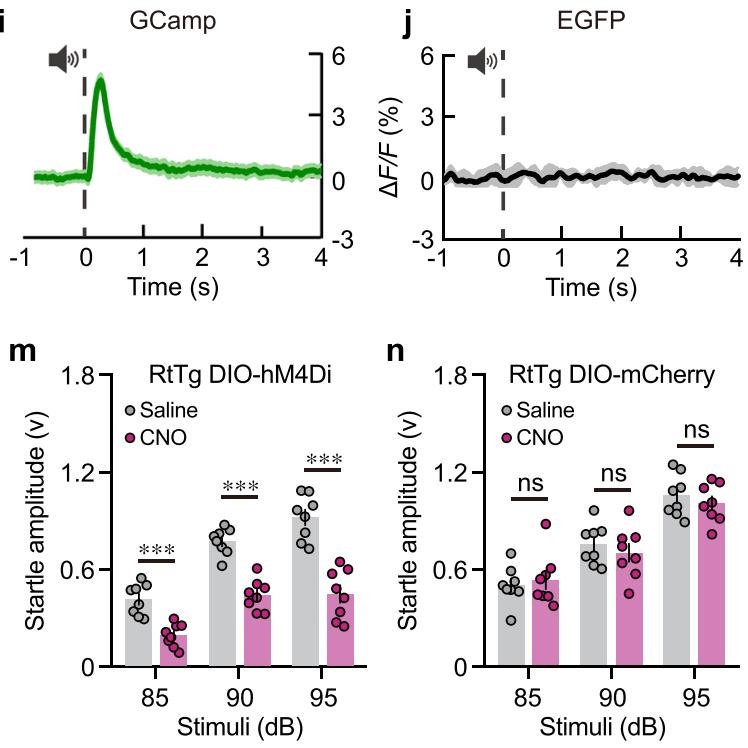

n

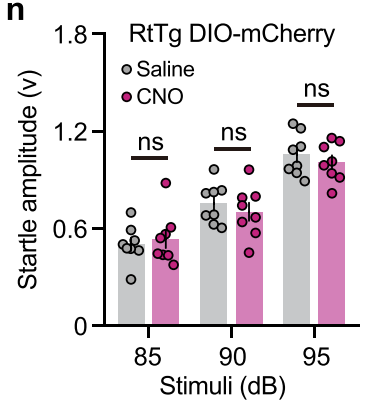

Fig. 3 RtTg glutamatergic neurons mediates ASR. a Schematic of ASR c-fos experiment. b Representative images showing control and $95 \mathrm{~dB}$ acoustic stimuli-induced c-fos expression in the RtTg. Scale bar, $200 \mu \mathrm{m}$. c Quantification of the c-fos ${ }^{+}$cell number in consecutive RtTg sections ( $n=6$ mice). AP, distance anterior or posterior to Bregma. $\mathbf{d}$ Left: a representative image showing RtTg c-fos ${ }^{+}$cells co-labelled with glutamate. Scale bar, $50 \mu \mathrm{m}$. Right: pie chart indicating the percentage of c-fos ${ }^{+}$neurons co-labelled with or without glutamate. e Schematic of fiber photometry setup. $\mathbf{f} A$ representative image confirming GCamp6m expression in the RtTg. Scale bar, $400 \mu \mathrm{m} . \mathbf{g}, \mathbf{h}$ Representative traces ( $\mathrm{g}$ ) and heat map (h) of calcium signal changes of RtTg glutamatergic neurons aligned to the acoustic stimuli onset. $\mathbf{i}, \mathbf{j}$ Quantifications of calcium (i, $n=35$ bouts from 5 mice) and EGFP (j, $n=25$ bouts from 5 mice) signal changes of RtTg glutamatergic neurons aligned to the acoustic stimuli onset. The thick line indicates the mean and the area shaded in lighter color indicates s.e.m. $\mathbf{k}$ The average peristimulus time histogram showing RtTg neuronal activities evoked by acoustic stimulus. I Schematic for chemogenetic inhibition of glutamatergic neurons in the RtTg of Vglut2-Cre mice. $\mathbf{m}, \mathbf{n}$ ASR amplitudes of Vglut2-Cre mice receiving bilateral injection of AAV-DIO-hM4Di-mCherry ( $\mathbf{m})$ or control AAV-DIO-mCherry $(\mathbf{n})$ into the RtTg, followed by i.p. administration of $\mathrm{CNO}$ or saline $(n=8$ per group; for m: $t=4.026, P=0.0002(85 \mathrm{~dB}) ; t=6.049, P=3.38 \times 10^{-7}(90 \mathrm{~dB}) ; t=8.626, P=7.63 \times 10^{-11}(95 \mathrm{~dB}) ;$ for $n: t=0.4851, P=0.6301(85 \mathrm{~dB}) ; t=0.7525$, $P=0.4559(90 \mathrm{~dB}) ; t=0.6869, P=0.4959(95 \mathrm{~dB}))$. All data are presented as the mean \pm s.e.m. Significance was assessed using two-way ANOVA combining with FDR corrections. ${ }^{\star \star \star} P<0.001$; ns, not significant.

RtTg receives direct projections from $\mathrm{CN}$. Given that the $\mathrm{CN}$ is the initial central auditory structure to receive input from the cochlear via the auditory nerve $\mathrm{e}^{30}$, our discovery that the RtTg mediates ASR motivated us to examine if the RtTg is a downstream target of the $\mathrm{CN}$. We conducted projection tracing experiments, wherein AAV-hSyn-EGFP ${ }^{31}$ was unilaterally injected into the $\mathrm{CN}$ (Fig. 4a). In addition to brain regions such as the lateral superior olivary nuclei (LSO) and the inferior colliculus (IC) known to receive direct $\mathrm{CN}$ inputs ${ }^{24,32}$ (Supplementary Fig. 16a-c), confocal images of serial coronal sections revealed dense $\mathrm{EGFP}^{+}$terminals in the RtTg (Fig. 4b and Supplementary
Fig. 17). Indicating a strong trend for cross-hemispherical projection, we noted that the EGFP signal was much stronger in contralateral RtTg than ipsilateral RtTg (Fig. 4c).

Next, we unilaterally injected the anterograde transsynaptic tracer AAV2/1-hSyn-Cre ${ }^{33}$ into the $\mathrm{CN}$ of Ai9 mice, a Cre-dependent tdTomato (tdT) reporter line ${ }^{34}$ (Fig. 4d). Consistent with our results from the aforementioned experiments with the AAV-hSyn-EGFP (Supplementary Fig. 16d, e), we detected abundant $\operatorname{tdT}^{+}$cells in RtTg, with especially dense labelling of neuron populations in the contralateral RtTg (Fig. 4e, f and Supplementary Fig. 18). Examining this RtTg population specifically, we noted that $40.1 \%\left(\mathrm{NeuN}^{+} \mathrm{tdT}^{+}\right.$ 
a

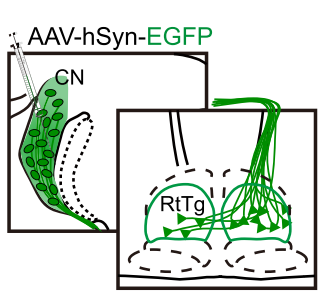

d

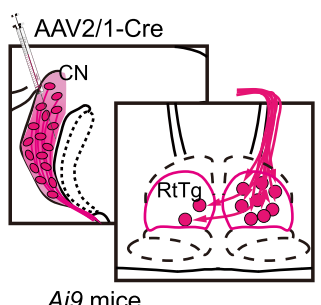

b
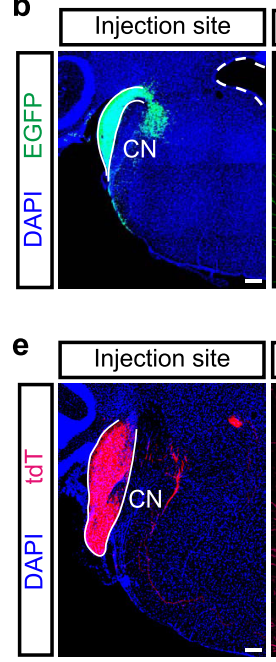
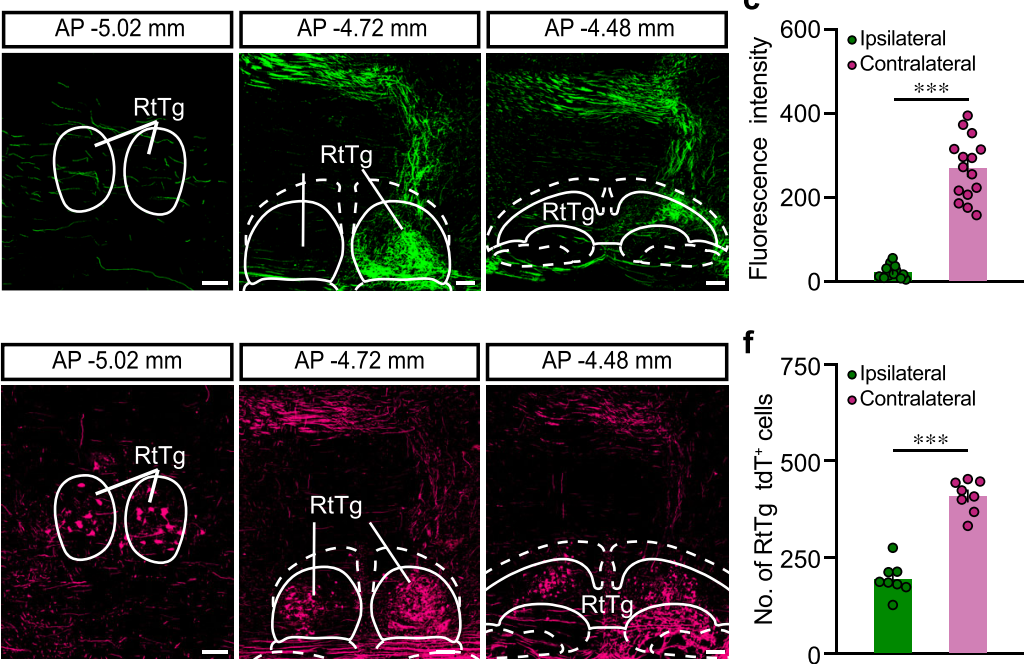

g
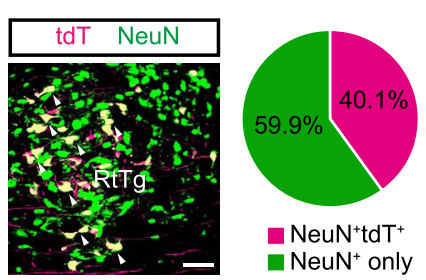

h

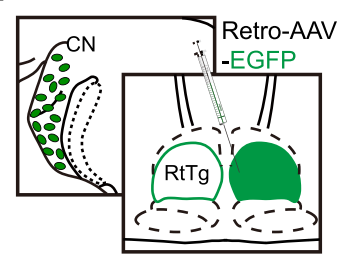

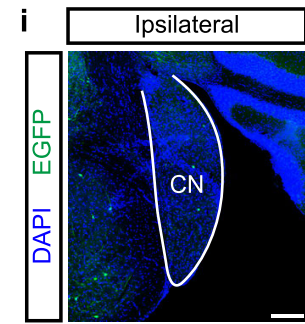
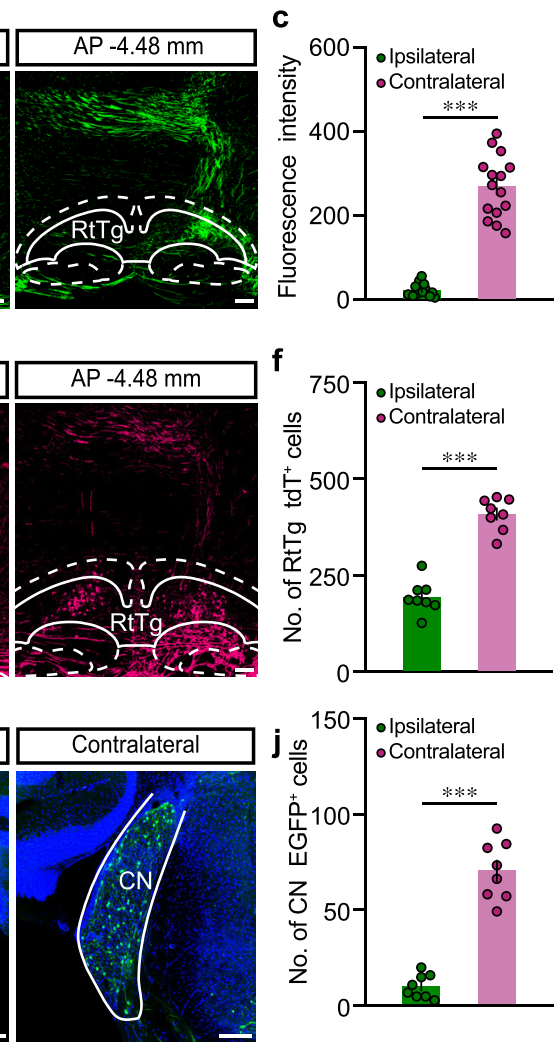

Fig. 4 Identification of a CN-RtTg direct projection. a Schematic for the anterograde tracing from CN to RtTg. b Representative images showing CN injection site and EGFP+ presynaptic terminals in RtTg. Scale bar, $200 \mu \mathrm{m}$. c Quantitative fluorescence intensity of CN EGFP ${ }^{+}$presynaptic terminals in the ipsilateral and contralateral RtTg $\left(n=15 ; P=8.87 \times 10^{-9}\right)$. d Schematic for the anterograde transsynaptic tracing from CN to RtTg. e Representative images showing CN injection site and the tdT+ cells in RtTg. Scale bar, $200 \mu \mathrm{m}$. f Quantification of tdT+ cell numbers in the ipsilateral and contralateral RtTg $\left(n=8 ; P=3.8 \times 10^{-7}\right)$. $\mathbf{g}$ Left: a representative image showing $\mathrm{tdT}^{+} \mathrm{RtTg}$ neurons co-labelled with NeuN (white arrowheads). Scale bar, $50 \mu \mathrm{m}$. Right: pie chart indicating the percentage of $\mathrm{NeuN}^{+}$neurons co-labelled with or without tdT. $\mathbf{h}$ Schematic for the retrograde tracing from RtTg to CN. $\mathbf{i}$ Representative images showing EGFP+ neurons in ipsilateral and contralateral CN. Scale bar, $200 \mu \mathrm{m}$. j Quantification of EGFP+ cells number in the ipsilateral and contralateral $\mathrm{CN}\left(n=8 ; P=5.09 \times 10^{-6}\right)$. Error bar represents mean \pm s.e.m. Significance was assessed using two-sided paired $t$-tests in $\mathbf{c}, \mathbf{f}, \mathbf{j}$. ${ }^{\star \star \star} P<0.001$.

cells) of total RtTg neurons received CN innervation (Fig. 4g and Supplementary Fig. 19).

To further confirm the existence of CN-RtTg projections, we unilaterally injected Retro-AAV-EGFP ${ }^{35}$ into the RtTg (Fig. 4h, Supplementary Fig. 20). Abundant $\mathrm{EGFP}^{+}$cells were observed in the $\mathrm{CN}$, with $\mathrm{EGFP}^{+}$cells number much higher in the contralateral $\mathrm{CN}$ than that in the ipsilateral $\mathrm{CN}$ (Fig. 4i, j). Together, these results demonstrate that the $\mathrm{CN}$ directly innervates the RtTg.

The glutamatergic $\mathrm{CN}-\mathrm{RtTg}$ projections mediate startle reflexes. To further investigate the nature of $\mathrm{CN}-\mathrm{RtTg}$ neural projections, we first tested whether the $\mathrm{CN}$ innervate RtTg glutamatergic neurons, which has been proved to mediate startle reflexes. Anterograde transsynaptic tracer AAV2/1-hSyn-Cre was unilaterally injected into the $\mathrm{CN}$ of $\mathrm{Ai} 9$ mice (Fig. 5a), and consecutive RtTg coronal sections were collected for glutamate immunostaining. As expected, nearly $90 \%$ of $\mathrm{tdT}^{+} \mathrm{RtTg}$ neurons contain glutamate, suggesting that $\mathrm{CN}$ neurons project extensively to RtTg glutamatergic neurons (Fig. 5b and Supplementary Fig. 21).

We next examined the type of neurotransmission between $\mathrm{CN}$ and RtTg by performing whole-cell patch-clamp recordings of the $\mathrm{RtTg}$ brain slices prepared from mice that received virallyencoded ChR2 injection into their CN (Fig. 5c). Photostimulation of $\mathrm{CN} \mathrm{ChR2} 2^{+}$terminals evoked excitatory postsynaptic currents (EPSCs) in most (6 of 7) RtTg neurons treated with Tetrodotoxin (TTX) and 4-aminopyridine (4-AP) ${ }^{36}$. Bath application of the glutamate receptor antagonist kynurenic acid (KYNA) completely blocked the light-evoked EPSCs (Fig. 5d). Collectively, these results demonstrate that $\mathrm{CN}$ neurons form monosynaptic glutamatergic synapses with RtTg neurons.

We then employed projection-based optogenetic-activation and chemogenetic-inactivation approaches to explore the functional contribution of this excitatory CN-RtTg innervation in freely moving mice. A Cre-dependent ChR2 or hM4Di virus was unilaterally/bilaterally injected into the $\mathrm{CN}$ of Vglut2-Cre mice, followed by implantation of an optical fiber or a guide cannula above the RtTg (Fig. 5e and Supplementary Fig. 22a, b). As expected, optogenetic activation of ChR2 terminals, but not EGFP terminals, in the RtTg elicited robust startle reflexes (Fig. 5f). On the contrary, chemogenetic inhibition of hM4Di terminals in the RtTg dramatically decreased ASR amplitudes (Supplementary Fig. 22c). These results further support that the CN-RtTg glutamatergic projections mediate ASR.

Spinal motor neurons are downstream targets of the RtTg. Given the above data indicating that optogenetic activation of the RtTg instantly evoked EMG activities in hindlimb muscles, we questioned whether the RtTg neurons specifically project to spinal motor neurons (MNs), as these neurons are known to mediate muscular movement. To define the segments of the spinal cord where RtTg neurons may terminate, we unilaterally injected the anterograde transsynaptic tracer AAV2/1-hSyn-Cre into the RtTg of Ai9 mice, followed by imaging of the spinal cord transverse sections from cervical to sacral segments (Fig. 6a). We observed 

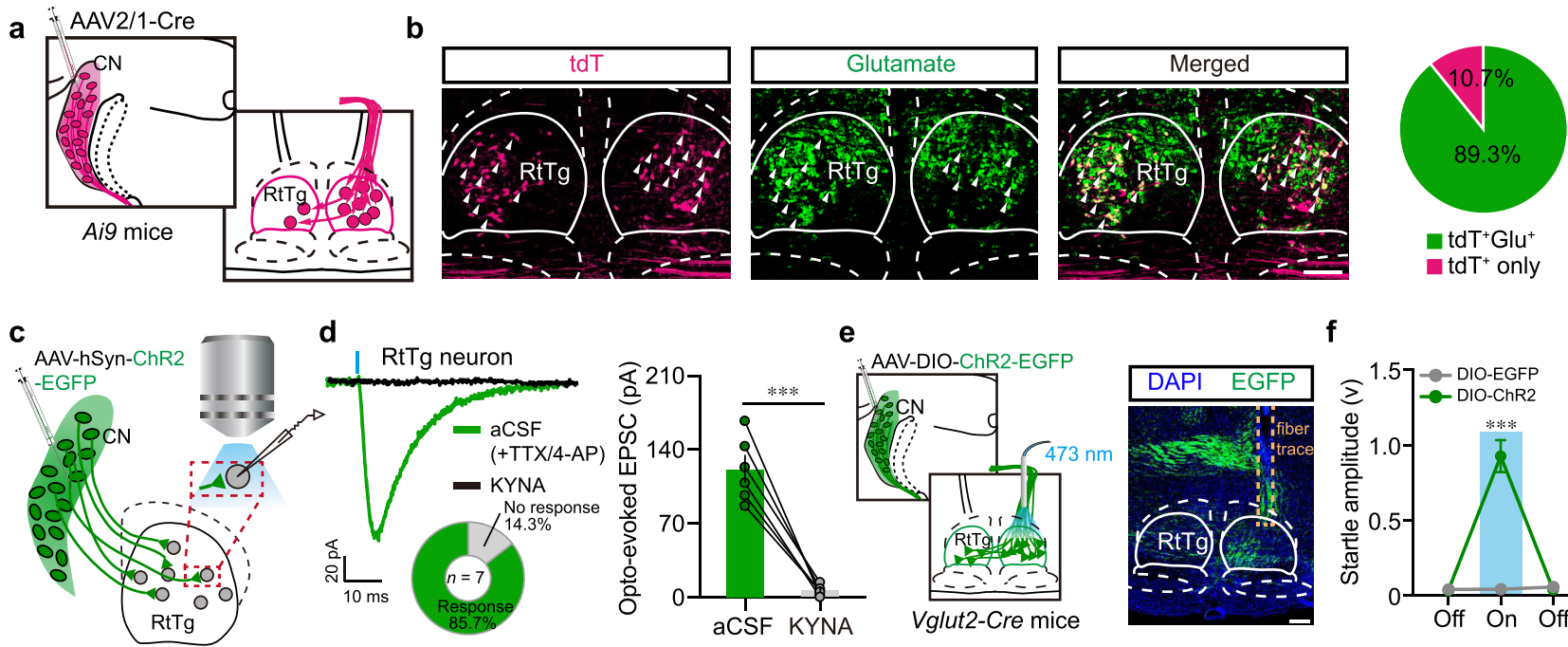

e
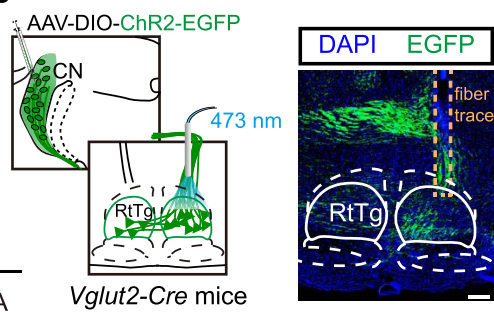

f

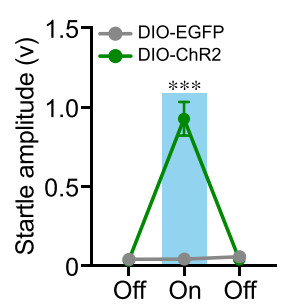

Fig. 5 The CN sends monosynaptic glutamatergic projections to RtTg glutamatergic neurons. a Schematic for the anterograde tracing from CN to RtTg. b Left: representative images showing RtTg tdT ${ }^{+}$neurons co-labelled with glutamate (white arrowheads). Scale bar, $300 \mu$ m. Right: pie chart indicating the percentage of RtTg tdT ${ }^{+}$neurons co-labelled with or without glutamate. c Schematic for the optogenetic activation of CN neuronal terminals and the

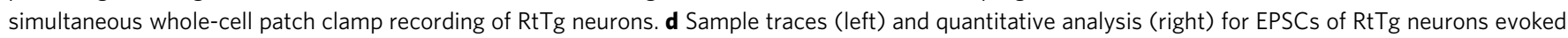
by photostimulation (blue bar, $1 \mathrm{~ms}$ ) before and after bath application of KYNA. TTX and 4-AP were added into aCSF ( $n=6$ cells from 4 mice;

$P=0.0003$ ). The doughnut chart shows the percentage of RtTg neurons responsive or not responsive to the photostimulation. $\mathbf{e}$ Left: schematic for the optogenetic activation of CN glutamatergic neuron terminals in the RtTg. Right: a representative image showing EGFP+ terminals in the RtTg. Scale bar, $300 \mu \mathrm{m}$. f Startle amplitudes evoked by photostimulation of ChR2 + terminals or EGFP + terminals in the RtTg of Vglut2-Cre mice $(n=15$ stimuli from 5 mice per group; $P=7.76 \times 10^{-7}$ ). Error bar represents mean \pm s.e.m. Significance was assessed using two-sided paired $t$-tests in d, and two-sided unpaired $t$-tests in f. ${ }^{\star \star \star} P<0.001$; ns, not significant.

that $\mathrm{tdT}^{+}$neurons are predominantly distributed in cervical and lumbar spinal cord segments, which have been previously demonstrated to harbor most of the neurons that innervate the limb muscles ${ }^{37,38}$. Immunostaining showed that these $\mathrm{tdT}^{+}$ neurons were co-labelled with an $\mathrm{MN}$-specific marker, choline acetyltransferase (ChAT) (Fig. 6b and Supplementary Fig. 23, 24).

Given previous studies reporting that some spinal interneurons also express $\mathrm{ChAT}^{39}$, to confirm that the RtTg indeed innervates muscle-controlling spinal MNs, we next performed rabies virusbased retrograde mono-transsynaptic tracing experiments ${ }^{40}$. Specifically, Retro-AAV encoding G protein and EGFP was injected into the hindlimb muscles of mice, followed by the injection of RV- $\Delta$ G-mCherry 3 weeks later (Fig. 6c). Using this approach we could specifically label the muscle-projecting spinal MNs. Starter MNs co-expressing EGFP and mCherry were found in the ventral horn of the spinal lumber segment (Fig. 6d). Furthermore, trans-synaptically labelled premotor neurons (expressing mCherry only) were abundantly found in the RtTg (Fig. 6d). These results indicate the existence of monosynaptic connections between RtTg and spinal muscle-controlling MNs.

To further examine whether the MN-projecting RtTg neurons also receive $\mathrm{CN}$ innervation, we unilaterally injected AAV2/1hSyn-Cre into the $\mathrm{CN}$ and AAV-DIO-EGFP-T2ASynaptophysin:mCherry ${ }^{41}$ into the contralateral RtTg (Fig. 6e). After confirming that transsynaptic spreading of Cre successfully labelled the RtTg neuron cytosol (EGFP) (Fig. 6f), we observed that the mCherry ${ }^{+}$presynaptic terminals of these RtTg neurons closely contact $\mathrm{ChAT}^{+}$spinal $\mathrm{MNs}$ in the spinal cervical and lumbar segments (Fig. 6g). Thus, we demonstrated a brainstem reticulotegmental neural ensemble that connects the auditory system with the motor functions, which mediates startle reflexes.

\section{Discussion}

Although sporadic reports from human and animal studies have revealed involvement of $\mathrm{RtTg}$ in smooth-pursuit eye movement as well as limb movements ${ }^{10-14}$, the research about RtTg is still very limited. In this study, we demonstrate that the RtTg acts as a startle-controlling nucleus. This conclusion is strongly supported by sufficient data from the present study: (1) Optogenetic activation of RtTg neurons elicited robust startle reflexes; (2) Chemogenetic inactivation and viral ablation of RtTg neurons suppressed startle amplitudes; (3) RtTg neurons exhibited increased calcium activities and firing spikes during ASR; (4) Activation of the RtTg induced EMG activities of neck and hindlimb muscles within millisecond latency, consistent with the nature of startle reflexes, a quick alert to sudden aversive stimuli.

Numerous early studies demonstrated that the PnC nucleus might play a critical role in startle reflexes ${ }^{17-19,42,43}$. According to the mouse brain atlas ${ }^{44}$, the RtTg situates closely adjacent to the PnC. Therefore, in this study, when manipulating the RtTg such as Casp3-based lesion, optogenetic activation as well as chemogenetic inhibition, we always checked whether there was any collateral influence on the PnC neurons, and any data with crosscontamination of the $\mathrm{PnC}$ were eliminated from the analysis. In other words, the identification of functional roles of the RtTg in the startle reflex should not be artifacts caused by destruction or stimulation of the adjacent $\mathrm{PnC}$ nucleus. Thus, based on previous and our studies, both the PnC and the RtTg are critically involved in mediating the startle reflex. In fact, the phenomenon that two different brain nuclei mediate the same behaviour does exists ${ }^{45,46}$. It is worth noting that in most previous studies, PnC neurons were activated by strong acoustic stimulus $(\geq 110 \mathrm{~dB})^{47-49}$, while in the present study, we used relatively lower acoustic stimulus (75-95 dB) to trigger the startle reflex and also observed the activation of RtTg neurons. Thus, it is possible that the $\mathrm{PnC}$ and the RtTg may have different sensitivities to acoustic stimulus patterns, such as intensity, frequency, duration, and sound type. Further investigation needs to be done to explore these possibilities and the potential structural as well as a functional connection between the RtTg and the PnC. 

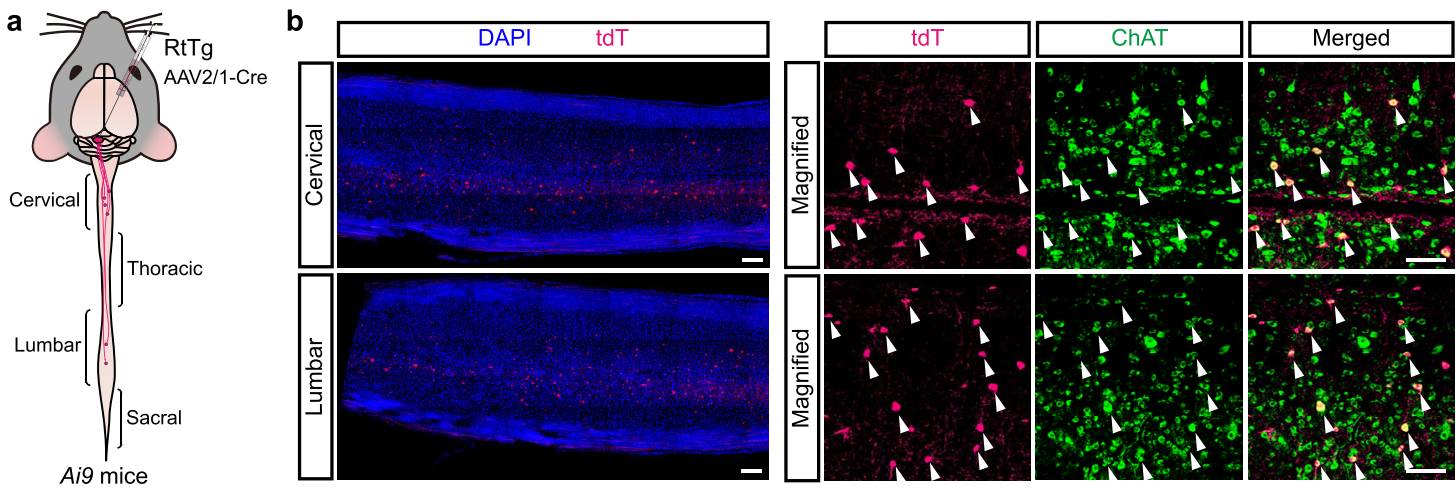

C
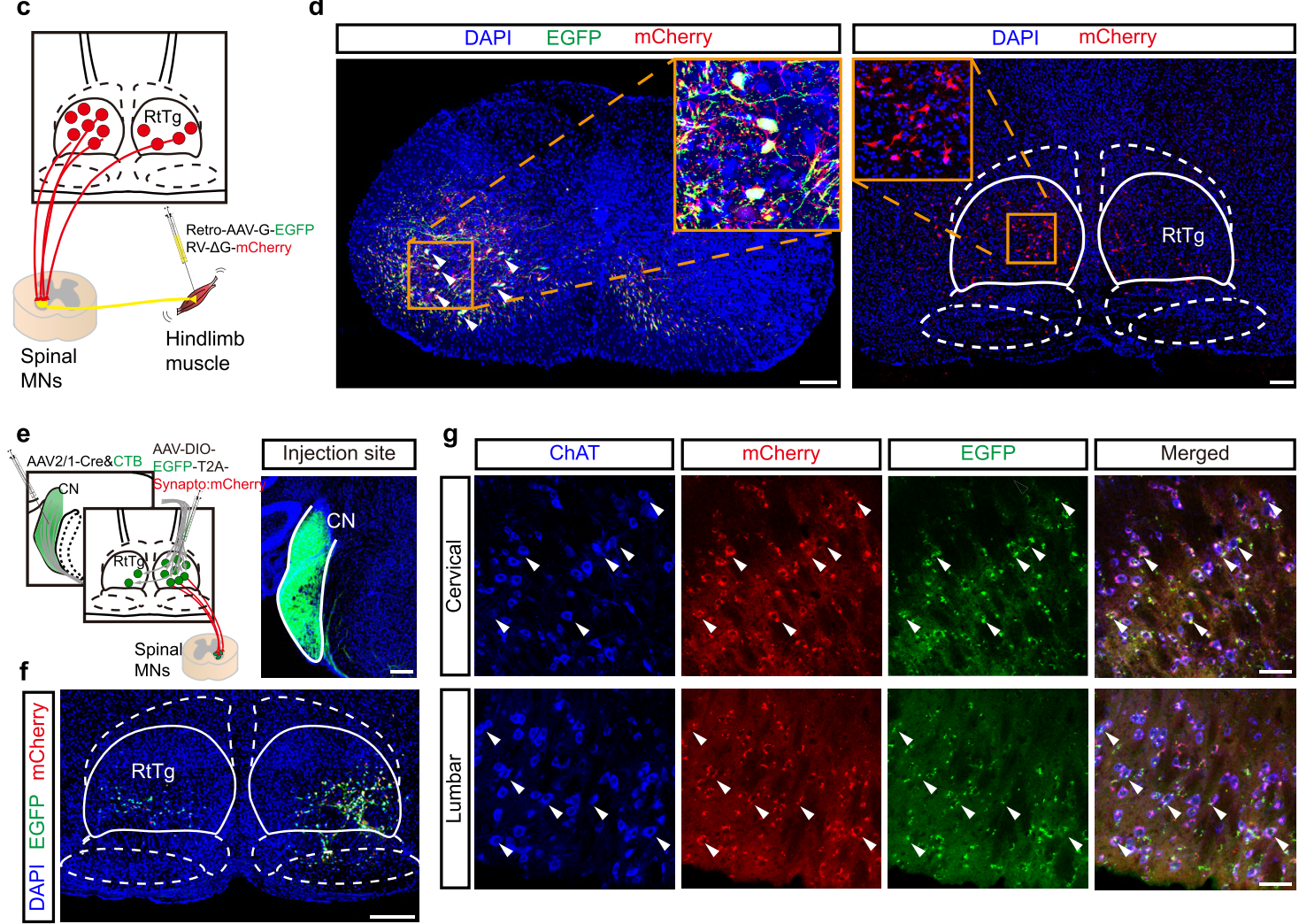

Fig. 6 Viral tracing of the CN-RtTg-MNs circuit. a Schematic for the AAV2/1-Cre-based anterograde transsynaptic tracing from the RtTg to multiple spinal cord segments. $\mathbf{b}$ Representative images showing the $\mathrm{tdT}^{+}$neuron labelling in transverse sections of spinal cervical and lumbar segments, and the co-labelling of tdT with ChAT (white arrowheads). Scale bar, $100 \mu \mathrm{m}$. c Schematic for rabies virus-based retrograde monosynaptic tracing from hindlimb muscles to the RtTg. d Representative images showing EGFP ${ }^{+} m$ Cherry ${ }^{+}$spinal starter MNs (left) and mCherry ${ }^{+}$neurons in the RtTg (right). Scale bar, $200 \mu \mathrm{m}$. e Left: schematic for the multilevel anterograde tracing from the CN to the spinal cord by injecting AAV2/1-Cre (mixed with CTB to visualize injection site) into the $\mathrm{CN}$, followed by the injection of AAV-DIO-EGFP-T2A-Synaptophysin:mCherry into the contralateral RtTg. Right: a representative image showing CN injection site. Scale bar, $200 \mu \mathrm{m}$. f A representative image showing Cre-dependent expression of EGFP in RtTg neuron cytosol. Scale bar, $300 \mu \mathrm{m}$. $\mathbf{g}$ Representative images showing co-existence (white arrowheads) of the mCherry ${ }^{+}$presynaptic puncta of RtTg neurons and the spinal cervical segment ChAT + MNs. Scale bar, $200 \mu \mathrm{m}$ (Cervical, Lumbar) and $5 \mu \mathrm{m}$ (Merged).

Although innate, startle reflexes can be modulated by fear emotional state, known as fear-potentiated startle (FPS $)^{50}$. The abnormally exaggerated startle reflex is a common feature across different fear-associated neuropsychiatric disorders including posttraumatic stress disorder, panic disorder, and specific phobia ${ }^{50-52}$. To date, however, circuit mechanisms for FPS remain unclear, and the therapeutic treatments to relieve this symptom are obscure. Previous studies indicate that the amygdala-PnC neural projections may engage in the modulation of the startle reflex by fear emotions. As a newly identified startle-controlling nucleus, the RtTg may also receive projections from fear-encoding upstream nuclei and contribute to the development of FPS. For instance, in the present study, when performing retrograde viral tracing from the RtTg, we did observe several fear-related upstream nuclei, such as the dorsal raphe nucleus and the lateral habenular nucleus (Supplementary Fig. 20). Thus, dissection of the circuit basis of RtTg-mediated startle reflex should provide insight for further study of the mechanism underlying abnormal exaggerated startle symptoms observed in many psychiatric disorders.

\section{Methods}

Animals. All animal experiments were conducted following protocols approved by the Institutional Animal Care and Use Committee of the University of Science and Technology of China (USTC). C57BL/6 J, Vglut2-Cre, Vgat-Cre, and GAD1-EGFP mice 
were purchased from Beijing Vital River Laboratory or Jackson Laboratories. Mice were group-housed (3-5 animals per cage) in a stable environment $\left(23-25^{\circ} \mathrm{C}\right.$ ambient temperature and $50 \%$ humidity). Mice were maintained in a 12 -hour light/dark cycle (light on at 7 a.m.) with free access to ad libitum chow food and water, and were allocated randomly to control and experimental groups. All experiments were performed on 8-week male mice to avoid potential sex as well as age differences. All experimental data were obtained by individuals blind to the experimental condition.

Acoustic startle reflex (ASR) test. After a $30 \mathrm{~min}$ period of acclimation to the testing room, mice were placed in a chamber within the darkened startle apparatus (MED Associate) for the ASR test. Broadband white noise were emitted from a high-frequency speaker mounted above the apparatus. A load cell platform under the chamber digitize the pressure generated by startled mice, and ASR amplitude was analyzed using Startle Reflex Software (MED Associate). After 5 min habituation period inside the startle chamber, mice received 30 startle trials (20-ms white noise stimuli with intensities of 85,90 , or $95 \mathrm{~dB}$ ). Each of the three white noise stimuli was applied 10 times in random order at random inter-trial intervals (ITIs) ranging from 58-63 s. The resulting startle reflexes of the mice in the startle chamber were recorded during $600 \mathrm{~ms}$ after acoustic stimuli onset. ASR amplitude of each trial was defined as the largest peak-to-trough response that occurred within $200 \mathrm{~ms}$ after the onset of the startle stimulus. ASR amplitude of each stimulus intensity was calculated as the mean amplitude of 10 startle trials.

Open field test. After a $30 \mathrm{~min}$ period of acclimation to the testing room, mice were individually placed into the open field area $\left(45 \times 45 \mathrm{~cm}^{2}\right)$ and their spontaneous motor activities were measured for $10 \mathrm{~min}$. The area was cleaned using $75 \%$ ethanol between each trial. Total distance together with time spent in the center zone were quantified automatically using ANY-maze system (Global Biotech Inc.).

Rotarod test. Before the training session started, mice were placed on the stationary rod for 5-min acclimation. Mice were trained three sessions per day for 2 days, with a $30 \mathrm{~min}$ inter-session interval. In each training session, mice were placed on the rotating rod accelerated from $4 \mathrm{rpm}$ to $60 \mathrm{rpm}$ in $300 \mathrm{~s}$. Total time was recorded (XR1514, Xinruan) until the mice fell off from the rod or gripped and circled around two times. Mice staying more than $300 \mathrm{~s}$ were taken manually from the rod, with total time recorded as $300 \mathrm{~s}$. After finishing the session, the apparatus was cleaned using $75 \%$ ethanol.

Tail-flick test. Tail-flick latency was measured using a tail-flick test apparatus (Ugo Basile). For each test, a radiant light beam was focused on a ventral portion of the mouse's tail ( $1.5-2 \mathrm{~cm}$ from its top). Light exposure was ended automatically once the mouse exhibit tail-flick motion. A 30 s cut-off time was employed to avoid skin injury. At the end of each test, the apparatus was cleaned with $75 \%$ ethanol alcohol.

Gait analysis. Gait analysis was conducted using the DigiGait imaging system (Mouse Specifics Inc.). Mice were placed individually on a transparent treadmill enclosed by a plexiglass compartment and have $3-5 \mathrm{~min}$ to freely explore the compartment while the treadmill remained still. After brief acclimation, the belt was started at an initial speed of $10 \mathrm{~cm} / \mathrm{s}$ and gradually increased to a constant speed of $25 \mathrm{~cm} / \mathrm{s}$. Digital videos of paw movement were acquired using a camera mounted under the treadmill. For each mouse, approximately $5 \mathrm{~s}$ of video were selected based on continuous movement. Videos were then analyzed using DigiGait software to automatically calculate gait parameters including stride-related variables as well as stance width.

Pupil monitoring. Mice were surgically prepared for head-fixed monitoring of pupil diameter. After a 3-day recovery period, mice were placed individually in a custom-made restrainer and were aroused using air-puff stimuli generated by a nitrogen tank connected to a solenoid-operated piston pump at random ITIs ranging from $60-80 \mathrm{~s}$. A small tube was placed behind the mice's head to deliver air puff. Pupil sizes were recorded using an infrared camera-based eye-tracking system (ISCAN systems, USA), filtered (CyberAmp, USA), and digitized (CED, UK) for offline analysis. \%changes of pupil diameter during air puff were calculated using custom-made MATLAB routines (The MathWorks, Natick, MA, USA).

Stereotaxic injection. Mice were anesthetized with an intraperitoneal injection of pentobarbital sodium $(0.5 \%, 0.01 \mathrm{ml} / \mathrm{g})$ and mounted on a stereotaxic apparatus (68015, RWD Life Science). Ophthalmic ointment was applied to prevent the eyes from drying. The skull was exposed by a midline scalp incision, and a craniotomy was drilled unilaterally or bilaterally above each cannula or optical fiber implantation or injection site. Viruses were delivered at a rate of $50 \mathrm{nl} / \mathrm{min}$ using glass pipettes connected to a pump (Legato130, KD Scientific). After completion of the injection, the glass pipettes were held in place for $10 \mathrm{~min}$ before being withdrawn to allow for diffusion of viruses. The implanted cannula or optical fiber was stabilized to the skull with dental cement. The animals were allowed to recover from anesthesia on a heating pad and housed for 2-4 weeks after surgery. Coordinates used for RtTg injection were as followed: bregma $-3.85 \mathrm{~mm}$, lateral $\pm 0.47 \mathrm{~mm}$, and dura $-4.75 \mathrm{~mm}$ (the actual infected RtTg region corresponds to AP $\sim-4.72 \mathrm{~mm}$ in the Mouse Brain Atlas, MBA). Coordinates used for $\mathrm{CN}$ injection were as followed: bregma $-5.35 \mathrm{~mm}$, lateral $\pm 2.45 \mathrm{~mm}$, and dura $-3.50 \mathrm{~mm}$ (the actual infected RtTg region corresponds to $\mathrm{AP} \sim-5.88 \mathrm{~mm}$ in the $\mathrm{MBA}$ ).

For manipulating the neuronal activity of RtTg in C57BL/6 J mice with optogenetics, rAAV-hSyn-hChR2(H134R)-EGFP-WPRE-pA (AAV2/9, $3.96 \times$ $10^{12} \mathrm{v} . \mathrm{g} . / \mathrm{ml}$, BrainVTA) was unilaterally injected into RtTg at a volume of $250 \mathrm{nl}$, followed by optical fiber implanted $0.2 \mathrm{~mm}$ above the injection site. rAAV-hSynEGFP-WPRE-pA (AAV2/9, $2.05 \times 10^{12} \mathrm{v} . \mathrm{g} . / \mathrm{ml}$, BrainVTA) was used as a control.

For chemogenetic inactivation of RtTg neurons, $100 \mathrm{nl}$ rAAV-hSyn-hM4DimCherry (AAV2/9, $6.42 \times 10^{12} \mathrm{v} . \mathrm{g} . / \mathrm{ml}$, Taitool) was bilaterally injected into RtTg of C57BL/6 J mice at a volume of $250 \mathrm{nl}$ for each side.

For ablating RtTg neurons, a 1:1 mixture of rAAV-hSyn-Cre-EGFP-WPRE-pA (AAV2/9, $3.21 \times 10^{12} \mathrm{v} . \mathrm{g} . / \mathrm{ml}$, BrainVTA) and rAAV-Efla-flex-taCasp3-TEVpWPRE-pA (AAV2/9, $5.80 \times 10^{12} \mathrm{v}$.g./ml, BrainVTA) or rAAV-EF1a-DIO-EGFPWPRE-pA (AAV2/9, $5.26 \times 10^{12} \mathrm{v} . \mathrm{g} . / \mathrm{ml}$, BrainVTA) was bilaterally injected into $\mathrm{RtTg}$ of C57BL/6 J mice at a volume of $250 \mathrm{nl}$ for each side.

To monitor the neuronal activity of RtTg glutamatergic neurons during startle, Vglut2-Cre mice were unilaterally injected with $250 \mathrm{nl} \mathrm{rAAV-Efla-DIO-Gcamp6m-}$ WPRE-pA (AAV2/9, $4.75 \times 10^{12} \mathrm{v} . \mathrm{g} . / \mathrm{ml}$, BrainVTA) into the RtTg. rAAV-EflaDIO-EGFP-WPRE-pA was used as a control.

For chemogenetic inhibition of RtTg glutamatergic neurons, rAAV-Ef1a-DIOhM4Di-mCherry-WPRE-pA (AAV2/9, $1.72 \times 10^{13} \mathrm{v}$.g./ml, Taitool) was bilaterally injected into the RtTg of Vglut2-Cre mice at a volume of $250 \mathrm{nl}$. rAAV-EF1a-DIOmCherry-WPRE-pA (AAV2/9, $5.26 \times 10^{12}$ v.g./ml, BrainVTA) was used as a control.

To dissert CN-RtTg projection, C57BL/6 J mice were unilaterally injected with $150 \mathrm{nl}$ rAAV-hSyn-EGFP-WPRE-pA (AAV2/9, $2.05 \times 10^{12} \mathrm{v} . \mathrm{g} . / \mathrm{ml}$, BrainVTA) into the $\mathrm{CN}$.

For anterograde transsynaptic tracing of CN-RtTg projection, Ai9 reporter mice were unilaterally injected with $150 \mathrm{nl}$ rAAV2/1-hSyn-Cre-WPRE-pA (AAV2/1, $1.03 \times 10^{13} \mathrm{v}$.g./ml, Taitool) into the $\mathrm{CN}$.

For optogenetic stimulation of CN-RtTg glutamatergic projection, rAAV-Ef1aDIO-hChR2(H134R)-EGFP-WPRE-pA (AAV2/9, $3.96 \times 10^{12}$ v.g./ml, BrainVTA) was unilaterally injected into $\mathrm{CN}$ of Vglut2-Cre mice at a volume of $150 \mathrm{nl}$, followed by optical fiber implanted $0.2 \mathrm{~mm}$ above the contralateral RtTg. rAAV-EF1a-DIOEGFP-WPRE-pA (AAV2/9, $5.26 \times 10^{12} \mathrm{v}$.g./ml, BrainVTA) was used as a control.

For chemogenetic inhibition of CN-RtTg glutamatergic projection, rAAV-Ef1 $\alpha$ DIO-hM4Di-mCherry-WPRE-pA was bilaterally injected into the CN of Vglut2Cre mice at a volume of $150 \mathrm{nl}$ for each side, followed by guide cannula bilaterally implanted above the RtTg. rAAV-EF1a-DIO-mCherry-WPRE-pA was used as a control.

For di-synaptic tracing of the $\mathrm{CN}-\mathrm{RtTg}-\mathrm{MNs}$ pathway, C57BL/6 J mice were unilaterally injected with $150 \mathrm{nl}$ rAAV2/1-hSyn-Cre-WPRE-pA (AAV2/1, $1.03 \times 10^{13} \mathrm{v} . \mathrm{g} . / \mathrm{ml}$, Taitool) into the CN and $250 \mathrm{nl}$ rAAV-Ef1 $\alpha$-DIO-mGFP-2ASynaptophysin-mCherry-WPRE-pA (AAV2/9, $4.54 \times 10^{11} \mathrm{v} . \mathrm{g} . / \mathrm{ml}$, BrainVTA) into the contralateral RtTg.

For retrograde tracing of RtTg-projecting neurons, C57BL/6 J mice were unilaterally injected with $250 \mathrm{nl}$ Retro-AAV-hSyn-EGFP-WPRE-pA (AAV2/R, $1.50 \times 10^{13}$ v.g./ml, Taitool) into the RtTg.

For retrograde monosynaptic tracing of spinal MNs-projecting premotor neurons, $2 \mu$ l helper virus (Retro-AAV-hSyn-G-EGFP-WPRE-pA, AAV2/R, $1.94 \times 10^{13} \mathrm{v} . \mathrm{g} . / \mathrm{ml}$, BrainVTA) was injected into the hindlimb muscles of $\mathrm{C} 57 \mathrm{BL} /$ $6 \mathrm{~J}$ mice. 3 weeks later, $1 \mu \mathrm{l}$ of the rabies virus RV-N2C- $\Delta$ G-mCherry $\left(4.72 \times 10^{7}\right.$ integral field units $/ \mathrm{ml}$ ) was injected into the same hindlimb muscles. 7 days later, mice were transcardially perfused and spinal as well as brain tissues were collected for the following imaging.

Unless otherwise specified, mice were group-housed for 3 weeks after viruses injection before further conducting behaviour tests or/and immunostaining. For all experiments after surgery, the mice with incorrect virus injections and fiber/ cannula implantation sites were excluded from analysis.

C-fos detection. To perform c-fos staining of the startle reflex, mice were placed in a darkened startle chamber for the ASR test. After a 5 min habituation period, mice received 10 startle trials (different fixed white noise intensities or air puffs were used in specific experiments). Each of the trials was applied at random inter-trial intervals (ITIs) ranging from 58-63 s. Mice of the control group were placed in the chamber for $15 \mathrm{~min}$ without white noise stimulation. $1.5 \mathrm{~h}$ after being placed in the startle chamber, mice were sacrificed for $\mathrm{c}$-fos immunostaining procedure.

Electromyography (EMG) recording. To perform EMG recording of neck and hindlimb muscles during optogenetic activation of RtTg neurons, AAV-hSynChR2-EGFP or AAV-hSyn -EGFP were unilaterally injected into the RtTg, followed by optical fiber implantation. Three weeks later, mice were mildly anesthetized with isoflurane (1-3\%), with the neck and hindlimbs were shaved off. The ends of the electrode wires were bared for $1-2 \mathrm{~mm}$ and threaded through surgical needles which were then inserted into the neck and hindlimb muscles. EMG signals were then amplified and filtered (Biotex Kyoto, Japan), digitized (Power 1401), and recorded (Spike2 Software) at a sampling rate of 1000-Hz. The Spike2 data were further analyzed using custom-made MATLAB routines. 
In vivo electrophysiological recordings. Mice were head-fixed in a stereotaxic frame in which the hollow ear bars were coupled to a white noise delivery system. After craniotomy and brain surface exposure, the linear silicon electrode (NeuroNexus, USA) was inserted into the RtTg to sample the neuronal firing rate of multiple units elicited by acoustic stimuli. Mice received startle trials with varied noise intensities $(60,75,80$, 85,90 , and $95 \mathrm{~dB}$ ). Each of the white noise stimuli was applied in random order at random inter-trial intervals (ITIs) ranging from 58-63 s. Signals were amplified, digitized (Blackrock, USA), and recorded (Nex Technologies, USA) at a sampling rate of $30 \mathrm{kHz}$. RtTg neuronal firing rate as well as firing latency after the onset of white noise were analyzed using NeuroExplore software (Plexon Inc.).

In vivo fiber photometry. Mice were housed individually for two weeks after AAV injection and optical fiber (200 $\mu \mathrm{m}$ diameter, 0.33 numerical aperture (NA), AniLab) implantation. Fluorescence signals were output to an amplifier (C7319, Hamamatsu) with a $0.01 \mathrm{~s}$ moving average filter (MF525-39, Thorlabs). The signals were then digitized (Power 1401) and recorded (Spike2 Software) at a $300 \mathrm{~Hz}$ sampling rate. The laser power at the tip of optical fiber was $15 \mu \mathrm{W}$ for RtTg. For monitoring the neuronal activity of RtTg during startle, we recorded white noise stimuli as well as airpuffs induced startle reflexes and $\mathrm{Ca}^{2+}$ transients or EGFP signals simultaneously. The raw data was then analyzed using MATLAB. $\mathrm{Ca}^{2+}$ fluorescence changes $(\Delta F / F)$ during startle reflex and conditioned fear were aligned to individual bouts and were calculated as $\left(\mathrm{F}-\mathrm{F}_{0}\right) / \mathrm{F}_{0}$, where $\mathrm{F}_{0}$ was the average fluorescence signals from $1 \mathrm{~s}$ preceding stimuli ( $95 \mathrm{~dB}$ stimuli or the $\mathrm{CS}$ ) to stimuli onset. $(\Delta F / F)$ values were presented as heatmaps or plotted with shaded area indicating s.e.m.

In vivo optogenetic manipulation. Mice were housed individually for three weeks after AAV injection and optical fiber ( $200 \mu \mathrm{m}$ diameter, $0.33 \mathrm{NA}$, AniLab) implantation. each optical fiber was attached to a 473-nm laser generator (AniLabOpto), and light pulses were delivered using Master-8 (A.M.P.I.). For in vivo optogenetic activation of RtTg neurons in general or RtTg glutamatergic neurons, mice were placed in a startle chamber and received blue light stimulation (473-nm, $15 \mathrm{~ms}$ pulse, $5 \mathrm{~mW}, 10 \mathrm{~mW}, 15 \mathrm{~mW}$, and $20 \mathrm{~mW}$ ). For activation of CN-RtTg projection, Vglut2-Cre mice were placed in the startle chamber and received blue light stimulation $(473 \mathrm{~nm}, 15 \mathrm{~ms}$ pulse, $15 \mathrm{~mW}$ ). Each mouse received 10 stimuli in total with an inter-trial interval of $30 \mathrm{~s}$, and opto-evoked startle amplitude was calculated as the mean amplitude of 10 stimuli trials.

Electrophysiological recording. Mice were anaesthetized deeply with an intraperitoneal (i.p.) injection of pentobarbital sodium $(0.5 \%, 0.01 \mathrm{ml} / \mathrm{g})$ and coronal brain slices $(300 \mu \mathrm{m})$ were sectioned using a vibratome (Leica VT1200S) after transcardially perfused with ice-cold modified $\mathrm{N}$-methyl-d-glutamine (NMDG) artificial cerebrospinal fluid (aCSF) which contained NMDG $93 \mathrm{mM}, \mathrm{KCl} 2.5 \mathrm{mM}, \mathrm{NaH}_{2} \mathrm{PO}_{4} 1.2 \mathrm{mM}$, $\mathrm{NaHCO}_{3} 30 \mathrm{mM}, \mathrm{N}$-2-hydroxyethylpiperazine-N-2-ethanesulfonic acid (HEPES) $20 \mathrm{mM}$, glucose $25 \mathrm{mM}$, Na-ascorbate $5 \mathrm{mM}$, Na-pyruvate $3 \mathrm{mM}$, thiourea $2 \mathrm{mM}$, $\mathrm{MgCl}_{2} 10 \mathrm{mM}$ and $\mathrm{CaCl}_{2} 0.5 \mathrm{mM}$ (continuously bubbled with $5 \% \mathrm{CO}_{2}$ and $95 \% \mathrm{O}_{2}$, $\mathrm{pH} 7.4,300-310 \mathrm{mOsm}$ ). Brain slices were incubated initially in NMDG aCSF (10 min, $32^{\circ} \mathrm{C}$ ) before transferred to HEPES aCSF that contained $\mathrm{NaCl} 92 \mathrm{mM}, \mathrm{KCl} 2.5 \mathrm{mM}$, $\mathrm{NaH}_{2} \mathrm{PO}_{4} 1.2 \mathrm{mM}, \mathrm{NaHCO}_{3} 30 \mathrm{mM}$, HEPES $20 \mathrm{mM}$, glucose $25 \mathrm{mM}$, Na-ascorbate $5 \mathrm{mM}$, Na-pyruvate $3 \mathrm{mM}$, thiourea $2 \mathrm{mM}, \mathrm{MgCl}_{2} 2 \mathrm{mM}, \mathrm{CaCl}_{2} 2 \mathrm{mM}$ (continuously bubbleed with 5\% $\mathrm{CO}_{2}$ and 95\% $\mathrm{O}_{2}, \mathrm{pH} 7.4,300-310 \mathrm{mOsm}$ ) for approximately $1 \mathrm{~h}$ at $21-23^{\circ} \mathrm{C}$. After recovery, slices were transferred into a recording chamber and continuously perfused with HEPES aCSF (continuously bubbled with $5 \% \mathrm{CO}_{2}$ and $95 \%$ $\mathrm{O}_{2}$ ). A fluorescent microscope (BX51WI, Olympus) with IR-DIC and a CCD camera was used to visualize target neurons.

For recording opto-evoked action potentials in $\mathrm{ChR} 2^{+}$neurons, whole-cell current clamp mode was obtained with pipettes (5-7 M 2 ) filled with internal solution which contained K-gluconate $145 \mathrm{mM}, \mathrm{MgCl}_{2} 4 \mathrm{mM}$, HEPES $10 \mathrm{mM}$, EGTA $10 \mathrm{mM}, \mathrm{Mg}$ ATP $5 \mathrm{mM}$, and Na-GTP $0.3 \mathrm{mM}$ (pH 7.2, 285-290 mOsm). $473 \mathrm{~nm}$ blue light ( $5 \mathrm{~mW} /$ $\mathrm{mm}^{2}, 5$ or $10 \mathrm{~Hz}$ ) was delivered to activate $\mathrm{ChR} 2^{+}$neurons.

Opto-evoked excitatory postsynaptic currents (EPSCs) were recorded with internal solution which contained K-gluconate $145 \mathrm{mM}, \mathrm{MgCl}_{2} 4 \mathrm{mM}$, HEPES $5 \mathrm{mM}$, EGTA $10 \mathrm{mM}$, Mg-ATP $5 \mathrm{mM}$, and Na-GTP $0.3 \mathrm{mM}$ (pH 7.2, 285$290 \mathrm{mOsm})(\mathrm{pH} 7.2,285-290 \mathrm{mOsm})$. Opto-evoked inhibitory postsynaptic currents (IPSCs) were recorded with internal solution which contained $\mathrm{CsCl}$ $120 \mathrm{mM}, \mathrm{MgCl}_{2} 4 \mathrm{mM}$, HEPES $10 \mathrm{mM}$, EGTA $10 \mathrm{mM}$, Mg-ATP $5 \mathrm{mM}$, and $\mathrm{Na}-$ GTP $0.3 \mathrm{mM}$. Neurons were holding at -70 and $0 \mathrm{mV}$ using a whole-cell voltageclamp for recording opto-evoked EPSC and IPSC respectively. Single optical stimulation $\left(5 \mathrm{~ms}, 10 \mathrm{~mW} / \mathrm{mm}^{2}\right)$ were delivered to ChR2 ${ }^{+}$terminals. $2 \mathrm{mM} \mathrm{KYNA}$ was added into the HEPES aCSF to block the opto-evoked EPSC. 2 mM KYNA and $20 \mu \mathrm{M}$ bicuculline were added to the HEPES aCSF to block the opto-evoked IPSCs.

To determine the excitability of target neurons, whole-cell current clamp mode $(I=0 p A)$ was obtained with pipettes (5-7 M $\Omega$ ) filled with internal solution which contained K-gluconate $145 \mathrm{mM}, \mathrm{MgCl}_{2} 4 \mathrm{mM}$, HEPES $10 \mathrm{mM}$, EGTA $10 \mathrm{mM}, \mathrm{Mg}$ ATP $5 \mathrm{mM}$, and Na-GTP $0.3 \mathrm{mM}$ (pH 7.2, 285-290 mOsm). Depolarizing current step $(1 \mathrm{~s},-20$ to $140 \mathrm{pA})$ was injected into the target neurons. CNO was added to the HEPES aCSF (continuously bubbled with $5 \% \mathrm{CO}_{2}$ and $95 \% \mathrm{O}_{2}$ ) for at least $10 \mathrm{~min}$ to act on $\mathrm{hM} 3 \mathrm{Dq}^{+} / \mathrm{hM}_{4} \mathrm{Di}^{+}$cell bodies or terminals. For membrane potential changes recording, $\mathrm{CNO}$ was bath applied for $10 \mathrm{~min}$ after $5 \mathrm{~min}$ stable baseline.
The electrophysiological signals were recorded via a MultiClamp 700B amplifier (Molecular Devices) equipped with Digidata $1550 \mathrm{~A}$ (Axon Instruments), digitized at $10 \mathrm{kHz}$, and filtered at $3 \mathrm{kHz}$. Data with a series resistance $>25 \mathrm{M} \Omega$ or changed by $>20 \%$ were discarded before analyzed using Clampfit 10.3 (Molecular devices, USA) and Minianalysis (Synaptosoft).

Immunofluorescence. Mice were anaesthetized deeply with an i.p. injection of pentobarbital sodium $(0.5 \%, 0.01 \mathrm{ml} / \mathrm{g})$ and transcardially perfused with ice-cold PBS $(0.1 \mathrm{M}, 20 \mathrm{ml})$ followed by $4 \%$ paraformaldehyde (PFA) dissolved in $0.1 \mathrm{M}$ PBS. Brains or spinal cord were removed and then post-fixed in $4 \% \mathrm{PFA}\left(4^{\circ} \mathrm{C}\right.$, overnight) before being transferred to $30 \%$ sucrose-PBS $\left(4^{\circ} \mathrm{C}, 2\right.$ days). Brains were then frozen sectioned (Leica CM 1860) into $40 \mu \mathrm{m}$ coronal slices and collected into PBS for immunostaining. Floating sections were then washed twice in PBS, incubated in $0.5 \%$ Triton X-100 for $20 \mathrm{~min}$, blocked with $10 \%$ goat or donkey serum for $40 \mathrm{~min}$ (room temperature), followed by primary antibodies incubation at $4{ }^{\circ} \mathrm{C}$ for at least $20 \mathrm{~h}$. Primary antibodies include anti-c-fos (1:1000, Abcam, ab190289), anti-NeuN (1:500, Millipore, MAB377, clone A60), anti-glutamate (1:500, Sigma, G6642), anti-ChAT (1:200, Millipore, MAB144P), anti-GFP (1:100, Thermo Fisher, A-11122). After being washed twice in PBS, sections were incubated with secondary antibodies for $1.5 \mathrm{~h}$ (room temperature) Secondary antibodies included goat anti-rabbit Alexa Fluor 488 (1:1000, Cell Signaling Technology, 4412 S), goat anti-rabbit Alexa Fluor 594 (1:1000, Cell Signaling Technology, 8889 S), goat anti-mouse Alexa Fluor 594 (1:1000, Thermo Fisher, A-11032), and Donkey anti-goat Alexa Fluor 488 (1:200, Thermo Fisher, A-11055). Finally, sections were stained with DAPI (1:1000, Sigma) before being mounted under coverslips with an anti-quenching mounting medium and imaged using Zeiss LSM 880 microscope. For $\mathrm{c}$-fos staining, mice were perfused $1.5 \mathrm{~h}$ after behavioural experiments. Cell numbers were counted automatically using Image $(\mathrm{NIH})$.

Drug infusion. For chemogenetic inhibition of RtTg neurons, C57BL/6 J/Vglut2Cre/Vgat-Cre mice were i.p. injected with CNO (5 mg/kg, Sigma) $30 \mathrm{~min}$ before conducting following behaviour test.

For chemogenetic inhibition of CN-RtTg projections, mice were housed individually for 1-4 weeks after AAV injection or/and cannula implantation. Internal injectors attached to $10 \mu \mathrm{L}$ syringes were inserted into guide cannula (RWD) for local CNO $(0.2 \mu \mathrm{g} / \mu \mathrm{l}, 500 \mathrm{nl} / \mathrm{side}$, Sigma) infusion at a rate of $200 \mathrm{nl} /$ min using an infusion pump (KDS Scientific). After the infusion, injectors were hold still for $1 \mathrm{~min}$ to allow for full drug diffusion into the target region. Behaviour tests were conducted $15 \mathrm{~min}$ after CNO infusion.

Statistics and reproducibility. Statistical analysis was performed using MATLAB (MathWorks) and GraphPad Prism 8.0 (GraphPad Software). Comparisons between control and treatment groups were conducted using paired/unpaired $t$-tests or two-way ANOVA combined with FDR corrections, as indicated in the figure legends. Data are presented as mean \pm s.e.m, with significant difference defined as $P<0.05$. No statistical methods were used to predetermine sample sizes, but our sample sizes are similar to those reported in previous publications. Unless otherwise noted, all representative experiments were repeated at least three times independently with similar results.

Reporting summary. Further information on research design is available in the Nature Research Reporting Summary linked to this article.

\section{Data availability}

The in situ hybridization database from Allen Mouse Brain Atlas (https://mouse.brain map.org/experiment/show/73818754) (https://mouse.brain-map.org/experiment/show/ 72081554) were used. The data that support the findings of this study are provided in the article and its Supplementary information files, and are available from the corresponding author upon reasonable request. Source data are provided with this paper.

\section{Code availability}

The custom codes that support the findings of this study are available from the corresponding author upon reasonable request.

Received: 26 May 2021; Accepted: 20 October 2021; Published online: 04 November 2021

\section{References}

1. Mangold, S. A. \& M Das, J. Neuroanatomy, Reticular Formation. StatPearls. (2020).

2. French, J. D. The reticular formation; the nature of the reticular activating system. J. Neurosurg. 15, 97-115 (1958). 
3. Krone, L. et al. Top-down control of arousal and sleep: fundamentals and clinical implications. Sleep. Med Rev. 31, 17-24 (2017).

4. Garcia-Rill, E. Disorders of the reticular activating system. Med Hypotheses 49, 379-387 (1997).

5. Jang, S. H., Chang, C. H., Jung, Y. J., Kim, J. H. \& Kwon, Y. H. Relationship between impaired consciousness and injury of ascending reticular activating system in patients with intracerebral hemorrhage. Stroke 50, 2234-2237 (2019).

6. Faraguna, U., Ferrucci, M., Giorgi, F. S. \& Fornai, F. Editorial: the functional anatomy of the reticular formation. Front Neuroanat. 13, 55 (2019).

7. Martins, I. \& Tavares, I. Reticular formation and pain: the past and the future. Front Neuroanat. 11, 51 (2017).

8. Esposito, M. S., Capelli, P. \& Arber, S. Brainstem nucleus MdV mediates skilled forelimb motor tasks. Nature 508, 351-356 (2014).

9. Paxinos, G. \& Mai, J. K. The human nervous system (Academic, New York), Ed 3 (2012).

10. Gamlin, P. D. \& Clarke, R. J. Single-unit activity in the primate nucleus reticularis tegmenti pontis related to vergence and ocular accommodation. $J$. Neurophysiol. 73, 2115-2119 (1995).

11. Matsunami, K. Neuronal activity in nuclei pontis and reticularis tegmenti pontis related to forelimb movements of the monkey. Neurosci. Res. 5, 140-156 (1987).

12. Zangger, P. \& Schultz, W. The activity of cells of nucleus reticularis tegmenti pontis during spontaneous locomotion in the decorticate cat. Neurosci. Lett. 7, 95-99 (1978)

13. Noebels, J. L. \& Sidman, R. L. Inherited epilepsy: spike-wave and focal motor seizures in the mutant mouse tottering. Science 204, 1334-1336 (1979).

14. Brudzyński, S. M. \& Mogenson, G. J. The role of the nucleus reticularis tegmenti pontis in locomotion: a lesion study in the rat. Brain Res Bull. 12, 513-520 (1984).

15. Rost, B. R., Schneider-Warme, F., Schmitz, D. \& Hegemann, P. Optogenetic tools for subcellular applications in neuroscience. Neuron 96, 572-603 (2017).

16. Boyden, E. S. et al. Millisecond-timescale, genetically targeted optical control of neural activity. Nat. Neurosci. 8, 1263-1268 (2005).

17. Koch, M. The neurobiology of startle. Prog. Neurobiol. 59, 107-128 (1999).

18. Yeomans, J. S. \& Frankland, P. W. The acoustic startle reflex: neurons and connections. Brain Res Rev. 21, 301-314 (1995).

19. Lee, Y., López, D. E., Meloni, E. G. \& Davis, M. A primary acoustic startle pathway: obligatory role of cochlear root neurons and the nucleus reticularis pontis caudalis. J. Neurosci. 16, 3775-3789 (1996).

20. Armbruster, B. N., Li, X., Pausch, M. H., Herlitze, S. \& Roth, B. L. Evolving the lock to fit the key to create a family of G protein-coupled receptors potently activated by an inert ligand. Proc. Natl Acad. Sci. USA. 104, 5163-5168 (2007).

21. Falls W. A. Fear-potentiated startle in mice. Curr Protoc Neurosci. Chapter 8 (2002).

22. Tellez, L. A. et al. Separate circuitries encode the hedonic and nutritional values of sugar. Nat. Neurosci. 19, 465-470 (2016).

23. Morgan, J. I., Cohen, D. R., Hempstead, J. L. \& Curran, T. Mapping patterns of c-fos expression in the central nervous system after seizure. Science 237, 192-197 (1987).

24. Asaba, A., Hattori, T., Mogi, K. \& Kikusui, T. Sexual attractiveness of male chemicals and vocalizations in mice. Front Neurosci. 8, 231 (2014).

25. Lissek, S. et al. Airpuff startle probes: an efficacious and less aversive alternative to white-noise. Biol. Psychol. 68, 283-297 (2005).

26. Liu, H. et al. Cellular differences in the cochlea of CBA and B6 mice may underlie their difference in susceptibility to hearing loss. Front Cell Neurosci. 13, 60 (2019).

27. Vong, L. et al. Leptin action on GABAergic neurons prevents obesity and reduces inhibitory tone to POMC neurons. Neuron 71, 142-154 (2011).

28. Li, Y. et al. Serotonin neurons in the dorsal raphe nucleus encode reward signals. Nat. Commun. 7, 10503 (2016)

29. Abe, H. et al. Epithelial localization of green fluorescent protein-positive cells in epididymis of the GAD67-GFP knock-in mouse. J. Androl. 26, 568-577 (2005).

30. Kaas, J. H. Evolution of nervous systems. (Academic, New York), Ed 2 (2017).

31. Mao, T. et al. Long-range neuronal circuits underlying the interaction between sensory and motor cortex. Neuron 72, 111-123 (2011).

32. Schofield, B. R., Motts, S. D., Mellott, J. G. \& Foster, N. L. Projections from the dorsal and ventral cochlear nuclei to the medial geniculate body. Front Neuroanat. 8, 10 (2014).

33. Zingg, B. et al. AAV-mediated anterograde transsynaptic tagging: mapping corticocollicular input-defined neural pathways for defense behaviours. Neuron 93, 33-47 (2017).

34. Madisen, L. et al. A robust and high-throughput Cre reporting and characterization system for the whole mouse brain. Nat. Neurosci. 13, 133-140 (2010).

35. Tervo, D. G. et al. A Designer AAV variant permits efficient retrograde access to projection neurons. Neuron 92, 372-382 (2016).

36. Petreanu, L., Mao, T., Sternson, S. M. \& Svoboda, K. The subcellular organization of neocortical excitatory connections. Nature 457, 1142-1145 (2009).
37. Kiehn, O. Locomotor circuits in the mammalian spinal cord. Annu Rev. Neurosci. 29, 279-306 (2006)

38. Sharma, K. \& Peng, C. Y. Spinal motor circuits: merging development and function. Neuron 29, 321-324 (2001).

39. Miles, G. B., Hartley, R., Todd, A. J. \& Brownstone, R. M. Spinal cholinergic interneurons regulate the excitability of motoneurons during locomotion. Proc. Natl Acad. Sci. Usa. 104, 2448-2453 (2007).

40. Murabe, N. et al. Higher primate-like direct corticomotoneuronal connections are transiently formed in a juvenile subprimate mammal. Sci Rep. 8, 16536 (2018).

41. Knowland, D. et al. Distinct ventral pallidal neural populations mediate separate symptoms of depression. Cell 170, 284-297 (2017). e18.

42. Lingenhöhl, K. \& Friauf, E. Giant neurons in the rat reticular formation: a sensorimotor interface in the elementary acoustic startle circuit? J. Neurosci. 14, 1176-1194 (1994).

43. Yeomans, J. S., Li, L., Scott, B. W. \& Frankland, P. W. Tactile, acoustic and vestibular systems sum to elicit the startle reflex. Neurosci. Biobehav Rev. 26, 1-11 (2002).

44. Paxinos, G. \& Franklin, K. B. J. The Mouse Brain in Stereotaxic Coordinates (Academic Press, 2001)

45. Han, W. et al. Integrated control of predatory hunting by the central nucleus of the amygdala. Cell 168, 311-324 (2017). e18.

46. Shang, C. et al. A subcortical excitatory circuit for sensory-triggered predatory hunting in mice. Nat. Neurosci. 22, 909-920 (2019).

47. Takahashi, K. et al. Neural circuits containing pallidotegmental GABAergic neurons are involved in the prepulse inhibition of the startle reflex in mice. Biol. Psychiatry 62, 148-157 (2007)

48. Moreno-Paublete, R., Canlon, B. \& Cederroth, C. R. Differential neural responses underlying the inhibition of the startle response by pre-pulses or gaps in mice. Front Cell Neurosci. 11, 19 (2017).

49. Zaman, T. et al. BK channels mediate synaptic plasticity underlying habituation in rats. J. Neurosci. 37, 4540-4551 (2017).

50. Boecker, L. \& Pauli, P. Affective startle modulation and psychopathology: Implications for appetitive and defensive brain systems. Neurosci. Biobehav Rev. 103, 230-266 (2019).

51. Morgan, C. A. 3rd, Grillon, C., Southwick, S. M., Davis, M. \& Charney, D. S Fear-potentiated startle in posttraumatic stress disorder. Biol. Psychiatry 38, 378-385 (1995)

52. Grillon, C., Ameli, R., Goddard, A., Woods, S. W. \& Davis, M. Baseline and fearpotentiated startle in panic disorder patients. Biol. Psychiatry 35, 431-439 (1994).

\section{Acknowledgements}

We acknowledge support from National Natural Science Foundation of China (Grants 91849206, 91649121, 91942315, 32121002, 92049304), the Strategic Priority Research Program of the Chinese Academy of Sciences (Grant XDB39050000), Key Research Program of Frontier Science (CAS, Grant No. ZDBS-LY-SM002), CAS Interdisciplinary Innovation Team (JCTD-2018-20), the Fundamental Research Funds for the Central Universities, USTC Research Funds of the Double First-Class Initiative (YD9100002001), CAS Project for Young Scientists in Basic Research (YSBR-013).

\section{Author contributions}

W.X. initiated, designed, and supervised the project; W.G. and S.F. conducted most of the experiments; D.X. conducted open field tests and rotarod tests; H.D. and Y.L. conducted the Electromyography recordings; G.X., Z.W. and Y.Z. conducted in vivo multi-unit electrophysiological recordings; Y.M. and T.X. conducted pupil monitoring; W.G. and S.F. analyzed data with assistance from H.D., G.X., Z.W. (Zhikun Wan), Z.W. (Zhen Wang) and W.X.; W.X., and W.G. wrote the manuscript.

\section{Competing interests}

The authors declare no competing interests.

\section{Additional information}

Supplementary information The online version contains supplementary material available at https://doi.org/10.1038/s41467-021-26723-9.

Correspondence and requests for materials should be addressed to Wei Xiong.

Peer review information Nature Communications thanks Peng Cao and the other, anonymous, reviewer(s) for their contribution to the peer review of this work.

Reprints and permission information is available at http://www.nature.com/reprints

Publisher's note Springer Nature remains neutral with regard to jurisdictional claims in published maps and institutional affiliations. 
(c) (i) Open Access This article is licensed under a Creative Commons Attribution 4.0 International License, which permits use, sharing, adaptation, distribution and reproduction in any medium or format, as long as you give appropriate credit to the original author(s) and the source, provide a link to the Creative Commons license, and indicate if changes were made. The images or other third party material in this article are included in the article's Creative Commons license, unless indicated otherwise in a credit line to the material. If material is not included in the article's Creative Commons license and your intended use is not permitted by statutory regulation or exceeds the permitted use, you will need to obtain permission directly from the copyright holder. To view a copy of this license, visit http://creativecommons.org/ licenses/by/4.0/.

(C) The Author(s) 2021 